\title{
Decussatio Pyramid and Optical Chiasm as an Interesting Example of Evolutionary Process Useful in Understanding Some Spinal Cord Phenomena?
}

\author{
Luisetto M ${ }^{1 *}$, Farhan Ahmad Khan ${ }^{2}$, Ahmed Yesvi Rafa ${ }^{3}$, Behzad Nili Ahmadabadi ${ }^{4}$ and Ghulam Rasool \\ Mashori $^{5}$
}

${ }^{1}$ Department of Applied pharmacologist, Independent Researcher, Italy

${ }^{2}$ Department of Pharmacology, Government Medical College, India

${ }^{3}$ Yugen Research Organization (YRO), Independent Researcher, Bangladesh

${ }^{4}$ Pharm D/PhD innovative Pharmaceutical product development specialist, USA

${ }^{5}$ Professor of Pharmacology, People University of Medical \& Health Sciences for Women, Pakistan

*Corresponding author: Luisetto M, Department of Applied pharmacologist,

Independent Researcher, Italy.

Received Date: April 04, 2019

Published Date: May 02, 2019

\begin{abstract}
From evolutionary pattern of invertebrates and vertebrates some interesting peculiarities can be easily Observed and some change in environmental world colonized produced strange anatomic configurations. In example decussatio piramidy in motors system. This kind of evolutionary process can be responsible of a sort of intrinsic weakness of a system to be deeply investigated to produce new hypothesis in some spinal cord conditions.
\end{abstract}

Keywords: Gasteropods; Brain; Evolution; Neural networks; Decussatio pyramidi; Brain nerve rotation; ALS; Neurodegeneration; Sports

\section{Introduction}

The medullary pyramids are paired white matter structures of the brainstem's medulla oblongata and contain motor fibers (cortico-spinal and corticobulbar tracts)-named the pyramidal tracts). The lower limit of the pyramids is marked when the fibers cross (decussate)themselves. About $90 \%$ of the fibers decussate and travel down the lateral cortico-spinal tract, the other $10 \%$ travels down the anterior cortico-spinal tract. Extrapyramidal tracts are motor tracts that do not cross the medullary pyramids. Car accident with brainstem injuries that affect the pyramids at the medulla oblongata. often result of dislocation at the occiput or spinal level C1. Injuries to the pyramids can be caused ALSO by the quick hyperextension of the neck (spine cervical region).

Hyper-extension of the neck can pull and tear the pyramids, with symptoms like weakness in all four limbs, difficulty swallowing, difficulty to speak. A bilateral infarction with ischemia in the medullary pyramids can produce in motor quadri-plegia. To the scope of this work is interesting first to observe some peculiar facts in evolution of life: Colonization of land from sea: all land organisms need to avoid drying-out and from microscopic size must create special structures to withstand gravity; are needed change in respiration and gas exchange systems. The reproductive systems cannot depend more on water to carry their eggs and sperm towards each other. the earliest evidence of land plants and animals dates back to 480 to $450 \mathrm{Ma}$, modern land ecosystems only appeared in the Late Devonian, about 390 to 360 Ma. In the new environment $\mathrm{O} 2$ is a potent oxidant agent and the accumulation in terrestrial atmosphere resulted from the development of photosynthesis over $3 \mathrm{Ga}$, in cyanobacteria (blue-green algae), first primitive oxygenic photosynthetic organisms.

Protective endogenous antioxidant enzymes and molecules but also the exogenous dietary antioxidants helped this new organism to prevent the oxidative damage. (essential trace elements in 
redox and antioxidant metallo-enzymes). In Land invertebrates colonization: New necessity related the movement (motor system) was needed. Animals had to change their feeding, excretory systems, and new internal fertilization of their eggs. the oldest known air-breathing seem to be Pneumodesmus (archipolypodan millipede) in Middle Silurian, $428 \mathrm{Ma}$. (with presence of spiracles, the openings to the tracheal systems).

Trace fossils from 450 Ma show the land invertebrates evolutionary pattern. The Arthropods were well pre-adapted to colonise the land, the jointed exo-skeletons provided protection against desiccation, new locomotion way not dependent on water environment. The earliest fossils of gastropods on land date from the Late Carboniferous period.

So is clear the role played by invertebrates (and polmonate gasteropods) in emerged land colonizing and it is a very interesting fact. Some new property is needed in the new environment: in example new modality of locomotion (motor system), preventing of disidratation (shell production) and New respiratory need (pulmonated organism) and many other vs acquatic organism. But Invertebrates for bilion of years was the only animals that populate the hearth, and first to colonize the emerged lands. In this group is interesting to observe in particular the gasteropods evolutionary pattern: The name gasteropods come from the greek Gaster, stomach and Poda, feet. Is possible to verify that due by the shell Conformation some organ and neuronal ganglia present a rotation since 180 grade (named anatomic torsion rotation hypotesys) this organism slither on their foot (a new locomotory function), they have an univalve shell generally with an antiorary rotation genetically determined. Characteristic of gasteropods is the asymmetry of their principal's organ (The nervous system if divided in central and periferic like other organism).

The gasteropods shell show a spiraliform logarithmic pattern (like frattalic pattern). The logarithmic spiralis was studied by 1638 Cartesio, develop with a fixed enlargement turn after turn like gasteropods shell or arietes horn. The shell is related to an accumulation of old a new part fitted to contain a growing organism that non change in form. But is interesting to obseve that In actual human being is possible to verify that in decussatio pyramidi there is a cross of the fibres.About $70 \%$ that make possible that the pyramid way form a left encefalous control the right body part. Other example of ancient torsion can be optical chiasma and the colon presentation? But this anatomic peculiarity can be a factor to take in consideration in some disease presentation like ALS? In this article are analyzed this specific factor to submit to researcher a global conclusion. to observe evolutionary pattern of nervous system from invertebrate to vertebrate can help in Better understand some physio-patohological process and a new light. A review related pathological anatomy in some neuronal brain and spinal cords circuits can be the right instrument. In evolutionary pattern related some gasteropods a cross nerve is present in nervous system (not in all gasteropods).

In this invertebrate due by their shell there is an organ torsion since 180 grades and cordonal nerve cross themselves like an 8 "chiastoneuria “. Form the greek term chīastós, cross +nêuros (nerve): observed in Mollusk Gasteropodi Prosobranchi (viscere and nerve rotation) (the gasteropods invertebrates for milion of years was the only animals on land). The earliest fossil of gasteropods on land date from late carboniferous. Artropodes were well pre adapted to colonize land, their exoskeleton provided protection against dessication, and means of locomotion not depending on water. Even if vertebrates not directly comes from gasteropodes (but from echinoderm?) some genes are conserved in invertebrates and vertebrates.

In examples regenerative genes involved in repair of damaged organ and limbs (in example in vertebrates like in anphibia,lizard, liver in humans). Similar structure to the 3 segmentation centre of vertebrates: anterior neural crest, limitanting intratalamic zone and istmic organizator are also present in sea warms specie Saccoglossus kowalevskii. gene Fgf8/17/18 gene omologus of geni Fgf8, Fgf17 e Fgf18 in vertebrates, sfrp1/5, hh e wnt1 are expressed in ectoderma of emicordate and control the ectoderma evolution in invertebrates but also in vertebrates.

Optical chiasma in human and decussatio pyramid can be related to an evolutionary genetic process started with some gasteropods or is due by environment adaptation? But in human opitcal chiasma there is a crossing of nervous fibres inside in rigid skull. Instead another structure named decussatio pyramid in spinal cord is in a less rigid bone box. The optic nerve fibres on the nasal sides of each retina corresponding to the temporal side of each visual field, the image is inverted) cross over (decussate) to the opposite side of the brain via the optic nerve at the optic chiasm (decussation of medial fibers).

The crossing over of optic nerve fibres at the optic chiasm allows the visual cortex to receive the same hemispheric visual field from both eyes. Overlay and processing these monocular visual signals allow the visual cortex to generate a binocular - stereoscopic vision. This crossing phenomena is an adaptive feature of frontally oriented eyes, founded mostly in the predatory animals requiring a more precise visual depth perception. (Prey animals, with laterally positioned eyes, have little binocular vision, with more complete crossover of visual signals). Beyond the optic chiasm, with crossed and uncrossed fibers, the optic nerves become optic tracts. The signals pass on to the lateral geniculate body, and to the occipital cortex (Figures 1 to 5 ).

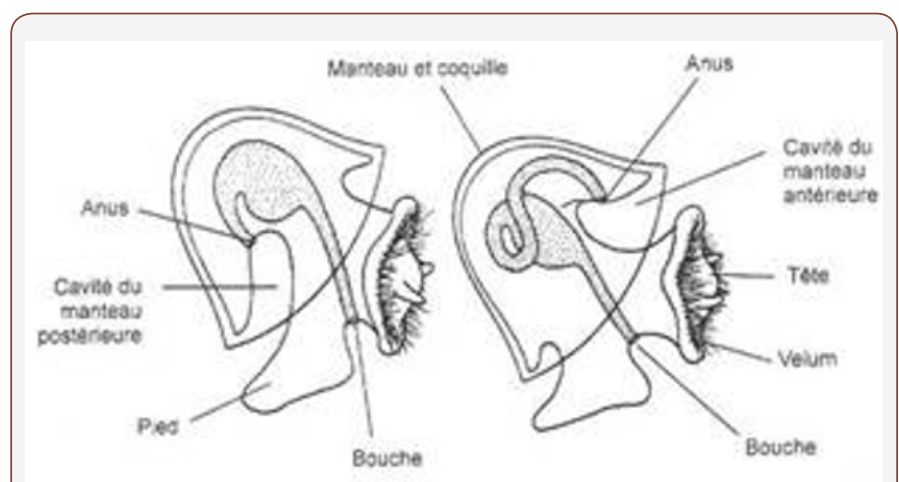

Figure 1: Flexion and torsion in Gasteropodes. 


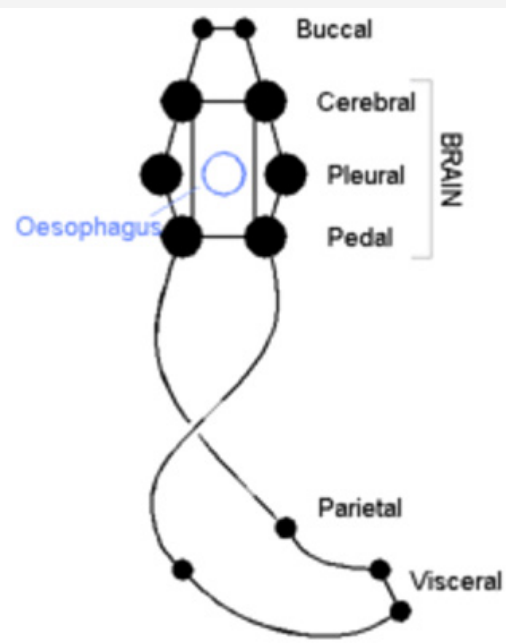

Figure 2: Nervous system in Gasteropodes (torsion).

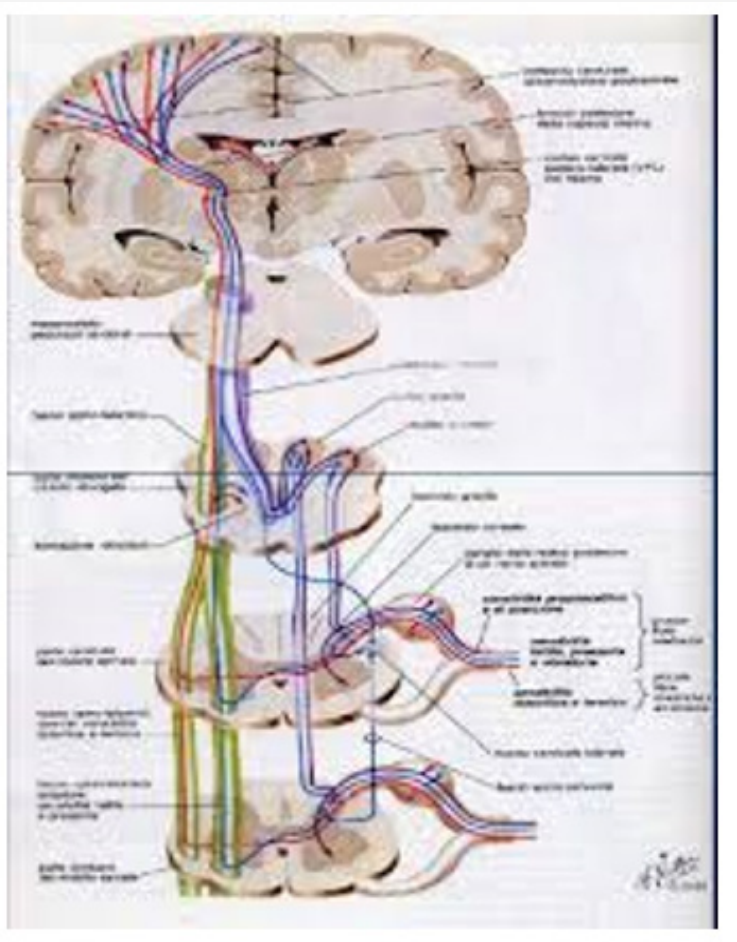

Figure 3: pyramidal way.

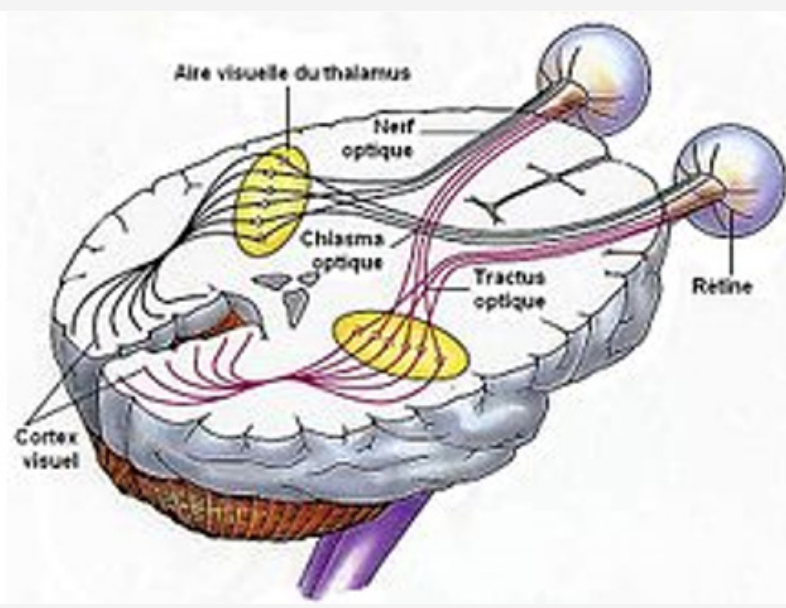

Figure 4: Optical chiasma.
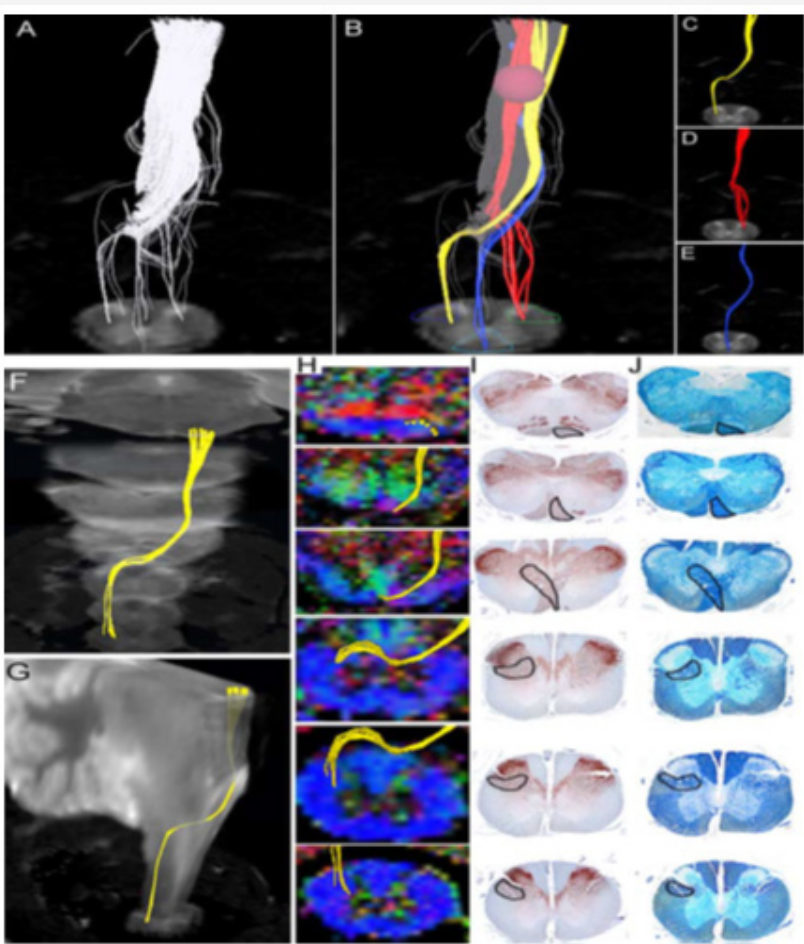

Figure 5: Pathway-specific DTT in a postmortem common marmoset revealing the course of the cortico-spinal tract with pyramidal decussation. A, DTT of the CST was conducted by placing the ROI in the pyramid of the medulla and tracing caudally.

The volume of traced tracts decrease as the tracing was carried caudally, many tracts became untraceable because of the partial volume effect. B, By placing secondary ROIs in areas of the upper cervical spinal cord known to contain CST fibers, CST fibers that pass through both ROIs could be depicted. C, Lateral CST fibers that crossed over to and descended the contralateral lateral funiculus in a pattern suggesting pyramidal decussation were depicted in yellow. D, Uncrossed lateral fibers descending the ipsilateral lateral funiculus were depicted in red. E, Lateral fibers descending the ipsilateral anterior funiculus were depicted in blue. DTT was capable of accurately depicting all threeknownpathways of the CST and this is significant. it is important to note that the depicted DTT tracts do not accurately reflect the volume of nerve fibers, because it is known that the lateral CST contains the majority of CST fibers. F, G, DTT of the pyramidal decussation superimposed on 3-dimensional MR images to macroscopically confirm that the pyramidal decussation was depicted in the proper height in the medulla and the upper cervical cord, using the cerebellum as a reference point.H, DTT of the pyramidal decussation super -imposed on axial color-coded FA maps. I, J, Axial histological slices of the same points in $\mathrm{H}$ stained for CaMKII- to reveal the location of CST fibers (I) and LFB to delineate the configuration of the white matter (J). In each slice, the area through which the DTT CST tract passes was positive for CaMKII- and LFB, confirming the accuracy of DTT. (from reference n. 24)

\section{Material and Methods}

With an observational approach some relevant scientific literature from PUBMED is analyzed in order to produce a global 
conclusion related the topics of this work. An experimental project is submitted to the researcher to support this conclusion

\section{Result}

From literature: according Louise R. Page "How did vertebrates get their head? How did tetrapods get their limbs? How did birds get their feathers? the need to explain the emergence of morphological novelties has been a major impetus for bringing developmental biology back within the embrace of evolutionary biology, because novelties can be difficult to explain under the gradualism of population genetics. to explaining the anterior mantle cavity in gastropods, this hypothesized rotation also accounted for the crossing of the pleuro-visceral nerve connectives ("streptoneury"), which is present in all but the most derived gastropod clades. The result has been a concept of gastropod torsion that I call the "rotation hypothesis": gastropods originated when all components of the visceropallium (shell, mantle, mantle cavity with contained structures, and viscera) rotated by $180^{\circ}$ relative to the head and foot. studies on representatives from 3 major clades of gastropods suggest that the highly conserved aspect of gastropod development is not synchronous rotation of all components of the viscera-pallium relative to the head and foot but rather a state of anatomical organization in which the developing mantle cavity is on the right but the shell coil is posterior (endogastric orientation). This conserved state of developmental anatomy has inspired an alternative hypothesis for the evolutionary origin of the gastropod body plan, the "asymmetry hypothesis": the gastropod mantle cavity originated from 1 side only of a bilateral set of mantle cavities. The asymmetry hypothesis does not require a saltation event to explain the origin of gastropods, nor does it require that the ancient molluscan precursor of gastropods carried the shell coil over the head (exogastric orientation torsion as a "grand controversy". A macromutation of the magnitude proposed by Garstang is difficult to accept. it is also difficult to imagine functional intermediate forms if the process of rotation occurred gradually, particularly if the shell participated in this rotation. From a starting condition (the hypothetical ancestral mollusk), the gastropod torsion is defined as a rotation by 180 of all components of the visceropallium relative to the cephalopodium. The rotation hypothesis has generated much controversy concerning the interpretation of Paleozoic molluscan fossils with a coiled shell. Debates focus on whether various extinct groups carried their coiled shell in an exogastric or in a endogastric orientation. literature on the evolution of gastropod torsion has not questioned whether the rotation actually occurred; rather it has debated the original adaptive value of the rotation. contemporary phylogenetic hypotheses do not place diotocardians (gastropods with 2 sets of pallial organs) as the most basal group of gastropods. Without independent evidence for a posterior mantle cavity in an ancient gastropod precursor, gastropod torsion is a "grand controversy" and a grand tautology." The single attempt to test experimentally any of these hypotheses found little support for Garstang's suggestion that ontogenetic torsion improves larval survival in the presence of planktivorous predators" [1].
Shuichi Shigeno et al: "Cephalopod and vertebrate neuralsystems are often highlighted as a traditional example of convergent evolution. Their large brains, relative to body size, and complexity of sensory-motor systems and behavioral repertoires offer opportunities for comparative analysis. Despite various attempts, questions on how cephalopod 'brains' evolved and to what extent it is possible to identify a vertebrate-equivalence, assuming it exists, remain unanswered. we summarize recent molecular/ anatomical / developmental data to explore certain features in the neural organization of cephalopods and vertebrates to investigate to what extent an evolutionary convergence is likely. based on whole body and brain axes as defined in early-stage embryos using the expression patterns of homeo domain-containing transcription factors and axonal tracto-graphy, we describe a critical analysis of cephalopod neural systems showing similarities to the cerebral cortex, thalamus, basal ganglia, midbrain, cerebellum, hypothalamus, brain stem, and spinal cord of vertebrates.

In squid, mantle contraction and jet propulsion are controlled by a giant fiber system consisting of 2 sets of 3 giant neurons organized in tandem. According to J. Z. Young and other, the "axons arising from the 2 first-order giant cells pass backward into the neuropil of the palliovisceral ganglion. Here they approach one another in the middle line and are joined by the inter-axonic bridge. The interest of this remarkable structure is that in the adult it consists not of a chiasma or crossing of 2 distinct fibers, but of a true protoplasmic bridge". Such an organization allows synaptic inputs from either side of the brain to be integrated and propagated down the giant fiber system as a symmetrical event for synchronous contraction of both sides of the mantle musculature. After the chiasm, these giant axons branch and establish synapses (chemical and electrotonicgap synaptic junctions) with several second-order giant axons in the neuropil of the palliovisceral lobe (SUB). From these cells, axons project from the central palliovisceral lobe (SUB) to the stellate ganglion in the mantle via the pallial nerve forming the presynaptic elements at the giant synapses. The axons of the giant system of cephalopods are thus part of an intricate network with other regions of the brain. Neural Structures Controlling Fast Escape Responses in Cephalopods, a Case of Analogy with the Hindbrain? Mauthner cells are one of the most historically notable motor systems for locomotory behavior reported in agnathans, teleost fish, and many amphibians. These are responsible for a rapid change in directionality and promote escape behavior. Mauthner neurons are characterized by a large neuronal cell, usually possessing a giant banana-shaped cell body located on either side of the midline in the brainstem with axon crossing to the contralateral spinal cord where they synapse with somato-motor neurons. The inputs to Mauthner neurons are primarily from receptors of the vestibular, auditory, and lateral line systems. In fish, the neurons are not the same size in all species, and this is considered to be linked to differences in taxa and possibly habitat "[2].

Larsson M: "The primate visual system has a uniquely high proportion of ipsilateral retinal projections, retinal ganglial cells 
that do not cross the midline in the optic chiasm. The assumption is that this developed due to selective advantage of accurate depth perception through stereopsis.the hypothesis that the need for accurate eye-forelimb coordination substantially influenced the evolution of the primate visual system is presented. Evolutionary processes may change the direction of retinal ganglial cells. Crossing or not, in the optic chiasm determines which hemisphere receives visual feed-back in reaching tasks. Each hemisphere receives little tactile and proprioceptive information about the ipsilateral hand. The eye-forelimb hypothesis proposes that abundant ipsilateral retinal projections developed in the primate brain to synthesize, in a single hemisphere, visual, tactile, proprioceptive, and motor information about a given hand, and that this improved eye-hand coordination and optimized the size of the brain. If accurate eyehand coordination was a great factor in the evolution of stereopsis, stereopsis is likely to be highly developed for activity in the area where the hands most often operate. The primate visual system is ideally suited for tasks within arm's length and in the inferior visual field, where most manual activity takes place. Altering of ocular dominance in reaching tasks, reduced cross-modal cuing effects when arms are crossed, response of neurons in the primary motor cortex to viewed actions of a hand, multi-modal neuron- response to tactile as well as visual events, extensive use of multimodal sensory information in reaching maneuvers support the premise that benefits of accurate limb control influenced the evolution of the primate visual system. The eye-forelimb hypothesis implies that evolutionary change toward hemi-decussation in the optic chiasm provided parsimonious neural pathways in animals developing frontal vision and visually guided forelimbs and suggests a new perspective on vision convergence in prey and predatory animals [3].

Kinsbourne M: "In the chordate and vertebrate central nervous system, sensory and motor nerve tracts cross from one side to the other as they connect the brain with sensory receptors and motor neurons. These "decussations," crossings in the form of an $\mathrm{X}$, relate each side of the brain to the opposite side of the body. The protochordates derive from an invertebrate ancestor, but no such contralateral arrangement occurs in any invertebrate phylum. No adaptive benefit of decussation has been established. What might explain the evolution of decussation? A brief review of relevant features of comparative morphology of invertebrates, chordates and vertebrates leads to an explanatory model of decussation.

A "somatic twist model" of invertebrate-vertebrate transition accounts for decussations as by-products of a more momentous change; the relocation of the neuraxis from the ventral to the dorsal aspect of the body. According Evidence this inversion proceeded by means of a twisting of the body 180 degrees on its axis just behind its anterior pole. This rotation aligned the neuraxis with the dorsal head ganglia and brain and by twisting the nerve tracts it brought decussation in its wake. Decussation evolved as a byproduct of a genetically determined partial inversion of the body plan, which resulted in a 180-degree rotation posterior to the brain and oropharynx [4].
De Lussanet MH et al: "One mysteries of the brain, which has puzzled all-time students of brain form and function, is the contralateral organization of the forebrain and the crossings of its major afferent and efferent connections. As a new explanation, 2 recent studies have proposed that the rostral part of the head, including the forebrain, is rotated by $180^{\circ}$ with respect to the rest of the body. Kinsbourne proposes one $180^{\circ}$ turn while we consider the $180^{\circ}$ being the result of two $90^{\circ}$ turns in opposite directions. We discuss the similarities and differences between the two hypothese" [5].

Troy Shinbrot et al: "Many vertebrate motor and sensory systems "decussate" or cross the midline to the opposite side of the body. The successful crossing of millions of axons during development requires a complex of tightly controlled regulatory processes. these processes have evolved in many distinct systems and organisms, it seems reasonable to presume that decussation confers a significant functional advantage-yet if this is so, the nature of this advantage is not understood. we examine constraints imposed by topology on the ways that a 3-dimensional processor and environment can be wired together in a continuous, somatotopic, way. as the number of wiring connections grows, decussated arrangements become overwhelmingly more robust against wiring errors than seemingly simpler same-sided wiring schemes. These results provide a predictive approach for understanding how 3D networks must be wired if they are to be robust, and therefore have implications both for future large-scale computational networks and for complex biomedical devices [6].

Marc Christopher Emos et al: A vast network of nerve tracts in the CNS which spans the cerebral cortex, brainstem, cerebellum, and spinal cord control the initiation and modulation of movements. The nerves in the CNS which carry the impulses for movement are known as upper motor neurons (UMN). The primary tract which carries signals for voluntary movement is known as the pyramidal tract. The pyramidal tract divides into the cortico-spinal tract and the cortico-bulbar tract. Injury or lesions to UMN's are common because of the vast areas covered by the motor neuron pathways. UMN lesions are designated as any damage to the motor neurons that reside above nuclei of cranial nerves or the anterior horn cells of the spinal cord. Damage to UMN's lead to a characteristic set of clinical symptoms known as the upper motor neuron syndrome. (symptoms can include weakness, spasticity, clonus, and hyper-reflexia). UMN's lesions have a wide differential diagnosis which ranges from cerebrovascular accidents, traumatic brain injury, malignancy, infections, inflammatory disorders, neurodegenerative disorders, and metabolic disorders.

Other Advanced neuro-imaging techniques. Structural MRI (sMRI) techniques enable detailed analysis of focal atrophy and regional grey and white matter. Typical high-resolution, singlecontrast images are 3-dimensional images acquired at 1-mm resolution in each dimension to ensure accurate structural demarcation. For some subcortical structures, tissue identification (segmentation) is best achieved through a combination of highresolution sequences In general, 2 classes of volume analysis 
are used: voxel-based morpho-metry (VBM), which measures relative grey and white matter volumes in specific brain regions; and surface-based morphometry (SBM), which measures cortical thickness.

Diffusion tensor imaging (DTI) quantifies Brownian motion of water molecules in biological tissues. By measuring both rate and preferred axis of water diffusion, DTI evaluate the integrity of white matter tracts, which can be degraded in neurological diseases. Fractional anisotropy (FA) is a scalar measurement of the degree of a preferred axis of water diffusion and decreases as the diffusion of water becomes less restricted to a single axis. Mean diffusivity (MD) ignores non-uniformity of the diffusivity and averages water diffusivity properties from all 3 axes. Additional diffusivity measures can be investigated using DTI, including radial diffusivity (diffusion perpendicular to the myelin sheath), and axial diffusivity (diffusion parallel to the myelin sheath). Data analysis can be performed using a region-of-interest approaches, which evaluate a specific brain location.

Whole-brain voxel-wise approaches such as tract-based spatial statistics; or tractography analyses, which can be used to characterize the white matter tracts. Proton magnetic resonance spectroscopy (1H-MRS) uses the slight differences in the magnetic field produced by electrons and nuclei in a localized region of tissue, to resolve and quantify certain tissue metabolites. The most commonly investigated metabolites are: $\mathrm{N}$-acetylaspartate (NAA), a marker of neuronal integrity; choline (Cho), a marker of cell membrane turnover; and creatine ( $\mathrm{Cr}$ ), a marker of energy metabolism. The Other brain metabolites include: myo-inositol, marker of glial cells; glutamate and glutamine (termed Glx); and GABA. The Metabolite quantification can be reported in the form of metabolite ratios or absolute concentrations. Metabolite ratios are used because standardization of the metabolite of interest relative to $\mathrm{Cr}$ or Cho corrects for variability that can arise, including magnetic field inhomogeneity and volume loss. Advances in 1H-MRS post-processing techniques, have facilitated absolute quantification of metabolites, considered to be more informative. Resting-state functional connectivity MRI (rs-fcMRI) uses intrinsic random fluctuations of brain activity to investigate relationships between brain regions. Detection of patterns of coherent brain activity led to the concept of specific neural networks in the brain.

Study demonstrated that, at rest, blood oxygen level-dependent signals (an indirect measure of neuronal activity) were synchronized between the motor cortices of each hemisphere, representing an intrinsic connection between these 2 cortical regions. Use of activation based functional MRI to elucidate neural correlates related to task execution-such as cognitive, motor and emotional tasks-demonstrated similar findings to the PET scientific literature. PET uses radioactive isotopes to localize and quantify brain metabolic activity or specific brain receptors, which can be altered in the disease processes. PET agents used to investigate CNS changes in ALS include 18F-fluorodeoxyglucose (FDG), a glucose analogue that is used to measure metabolism; 18F-6-fluorodopa, a dopamine precursor; 11C-PK11195, which binds to the translocator protein and is considered a marker of neuro-inflammation; 11C -flumazenil, which binds to the GABAA receptor; 11C-WAY100635, which binds to the serotonergic 5 -hydroxytryptamine receptor $1 \mathrm{~A}$; and 11C-L-deprenyl, which binds to monoamine oxidase B. Upper motor neurons are first-order neurons which are responsible for carrying the electrical impulses that initiate and modulate movement. Various descending UMN tracts are responsible for the coordination of movement. The major UMN tract that initiates voluntary movement is the pyramidal tract. The pyramidal tract provides a direct pathway between the cerebral cortex and the spinal cord, in contrast with extra-pyramidal tracts which provide indirect pathways for the coordination of movement. The pyramidal tract divides into the cortico-spinal tract and the cortico-bulbar tract. Cortico-spinal tract fibers synapse with spinal nerves while cortico-bulbar fibers synapse with cranial nerves. The cell bodies of the pyramidal tract concentrate around the motor area of the cerebral cortex. In general, the motor areas of the left and right hemispheres will innervate the musculature on the contralateral side of the body. The motor areas are somato-topically organized.

Upper motor neurons of the pyramidal tract have the majority of their cell bodies located in the pre-central motor cortex (Brodmann area 4) and the premotor area (Brodmann area 6). Cell bodies are also present in the supplementary motor area, primary somatosensory cortex, and the superior parietal lobe. UMN axons radiate out into the corona radiata and converge at the posterior limb of the internal capsule. The pathway of the cortico-spinal tract descends through cerebral peduncle in the midbrain, ventral pons, and the pyramids of the medulla. At the inferior aspect of the medulla, the majority of cortico-spinal tract axons decussate at the pyramidal decussation. The axons continue their descent contralateral from their cell bodies of origin and enter the spinal cord at the lateral funiculus. The tract is now the lateral cortico-spinal tract. The axons terminate throughout the spinal cord in the ventral gray column and the base of the dorsal column. The lateral corticospinal tract axons that control distal extremities synapse directly on lower motor neurons. These direct connections are presumed to be necessary for the fine control of the fingers and hands. The rest of the lateral cortico-spinal tract axons will synapse on premotor interneurons. At the pyramidal decussation, approximately $10 \%$ of the cortico-spinal tract axons do not decussate and continue their descent down the brain ipsilateral to their cell bodies of origin. These fibers enter the ventral aspect of the spinal cord and are known as the anterior cortico-spinal tract. As the fibers descend the spinal cord, most of them will decussate through the anterior white commissure before synapsing with inter-neurons. A small percentage of cortico-spinal axons will not decussate anywhere along their descent the brainstem or the spinal cord. These axons provide the impulses which control axial musculature necessary to maintain body posture.

The cortico-bulbar tract fibers originate from the parts of the motor cortex that represent the face. The axons share a similar trajectory to the cortico-spinal tract descending through the corona radiata and the internal capsule. At the level of the brainstem, the 
axons will synapse with each cranial nerve nuclei at their respective levels. The upper motor neuron innervation of most cranial nerves is bilateral which means that each cranial nerve receives impulses from the left and right hemisphere.

This bilateral innervation pertains to the muscles of the eyes, jaw, pharynx, upper face, larynx, and neck. 2 exceptions to this rule are CN VII and XII. The control of tongue protrusion by CN XII and the control of the muscles of the lower face by CN VII only receive contralateral innervation from the pyramidal tract. Knowledge about of pyramidal tracts is paramount to understanding the clinical presentation of UMN lesions. Lesions above or below the pyramidal decussation will have symptoms on different parts of the body. UMN lesions rostral to the pyramidal decussation will result in symptoms contralateral to the site of the lesion. For example, a unilateral lesion on the right cortico-spinal tract before the pyramidal decussation would cause weakness and spasticity of musculature on the left side of the body. UMN lesions caudal to the decussation will cause symptoms ipsilateral to the site of the lesion. This presentation would generally be with lesions to the spinal cord. left-sided lesions of the cortic-ospinal tract in the spinal cord will cause left-sided weakness and spasticity. Unilateral UMN lesions innervating cranial nerves do not manifest with clinically significant symptoms due to their bilateral innervation from the left and right motor areas. Hence, only bilateral lesions to the UMN of cranial nerves would create deficits. Lesions of UMN's to CN VII and $\mathrm{XII}$ are the exceptions because of their unilateral innervation from contralateral motor areas. a right-sided UMN lesion to the motor area that controls CN VII would manifest as a weakness on the left lower face.

A detailed patient history and a complete physical exam are essential for differentiating the cause of UMN lesions. Some diseases that can damage upper motor neurons include cerebrovascular accidents, amyotrophic lateral sclerosis, primary lateral sclerosis, multiple sclerosis, Brown-Sequard Syndrome, vitamin B12 deficiency. Cerebrovascular accidents or strokes are the sudden cessations of blood flow to areas of the brain leading to cell death. Strokes fall into 2 etiologic categories, being either ischemic or hemorrhagic. Ischemic strokes are the sudden interruption of blood flow to the brain which can be due to thrombi, emboli, or compression. Hemorrhagic strokes are characterized by bleeding into the brain due to rupture of a blood vessel. The cerebral blood supply has many branches which supply different areas of the brain. Occlusion of the middle cerebral artery or anterior cerebral artery can damage the motor areas of the cerebral cortex. Given the extensive areas of the brain supplied by the middle cerebral and anterior cerebral artery, strokes in those regions are likely to present with sensory, language, perceptual, and visual deficits in addition to UMN signs. Occlusion of the lenticulostriate arteries can damage the internal capsule. A stroke that targets the posterior limb of the internal capsule presents with pure motor deficits of the contralateral face, arm, and leg. Occlusion of the various branches of the vertebral artery or basilar artery can lead to strokes in different areas of the brainstem. Notable brainstem strokes that damage the cortico-spinal tract are medial medullary syndrome, medial pontine syndrome, and Weber Syndrome.

Amyotrophic lateral sclerosis (ALS) is the most prevalent neruo-degenerative disease that is characterized by its involvement of both upper and lower motor neurons. the clinical presentation is a combination of upper motor signs and lower motor neuron signs. Nerve conduction studies and electromyography are utilized to confirm the diagnosis. Labs are generally used to rule out other disease processes that can manifest with weakness in patients. ALS is currently incurable various treatments have been developed to extend life in patients. Riluzole is a glutamate pathway antagonist that is the only current drug shown to extend life in patients with ALS. Primary lateral sclerosis (PLS) is a neruo-degenerative disorder that targets upper motor neurons. PLS is generally seen in adults and is sporadic in nature, though hereditary variants have been observed. Compared to ALS, PLS has a slower progression and lacks lower motor neuron signs. some individuals with PLS do develop lower motor neuron signs as their disease progresses. The condition would then be considered upper motor neuron onset ALS. There are no cures for PLS and treatment is aimed at alleviating symptoms of spasticity and weakness through medications and physical therapy.

Brown-Sequard Syndrome is a spinal cord lesion caused by a hemisection injury to the spinal cord. The most common etiology is from penetrating trauma to the spine. other etiologies include blunt trauma, hematoma, tumors, or disc herniation. As a result of the hemisection of the spinal cord, the symptoms are manifestations of damage to the lateral cortico-spinal tract, dorsal column, and the lateral spino-thalamic tract. Patients present with upper motor neuron signs ipsilateral and below the level of the lesion. Patients will also present with ipsilateral loss of fine touch, vibration, and proprioception in addition to the contralateral loss of pain and temperature sensation.

Multiple Sclerosis (MS) is an immune-mediated, inflammatory demyelinating disease. The symptomatology of MS is characterized by episodes that occur in different anatomic locations in the central nervous system and occur months or years apart. The presenting symptoms of patients are highly variable. Symptoms can include cognitive disturbance, visual changes, hemiparesis, ataxia, and sensory deficits. The UMN signs of MS are due to the demyelination of upper motor neurons. MRI is the imaging test of choice used to diagnose MS. CSF studies may also be used to aid in diagnosis. Oligoclonal bands and intrathecal immunoglobulin G are classically seen in the CSF of MS patients. The most prevalent etiologies of vitamin B12 deficiency are pernicious anemia, bariatric surgery, small intestine surgery, and gastritis. Other etiologies include pancreatic insufficiency, inadequate dietary intake, and drug side effects. Vitamin B12 deficiency causes degeneration of the dorsal column and lateral white matter of the spinal cord. This can lead to degeneration of the lateral cortico-spinal tract with subsequent UMN signs. Degeneration of the dorsal column manifests as sensory ataxia. Deficiency also leads to macrocytic anemia. Supplementation 
with vitamin B12 generally corrects the anemia and stops the progression of degeneration of the spinal cord [7].

Iwata NK: A reliable objective marker of upper motor neuron (UMN) involvement is critical for early diagnosis and monitoring disease course in patients with amyotrophic lateral sclerosis (ALS). Lower motor neuron (LMN) involvement can be identified by electromyography, whereas UMN dysfunction has been currently distinguished solely by neurological examination. In the search for diagnostic tests to evaluate UMN involvement in ALS, numerous reports on new markers using neurophysiological and imaging techniques are accumulating. Trans-cranial magnetic stimulation evaluates the neurophysiological integrity of UMN. Although the diagnostic reliability and sensitivity of various parameters of central motor conduction measurement differ, central motor conduction time measurement using brainstem stimulation is potentially useful for determining UMN dysfunction by distinguishing lesions above the pyramidal decussation. MR-based techniques also have the potential to be used as diagnostic markers and are continuously improving as a modality to pursue early diagnosis and monitoring of the disease progression. Conventional MRI reveals hyperintensity along the cortico-spinal tract, hypointensity in the motor cortex, and atrophy of the precentral gyrus. There is a lack of agreement regarding sensitivity and specificity in detecting UMN abnormalities. Recent advances in magnetizing transfer imaging (MTI) provide more sensitive and accurate detection of corticospinal tract abnormality than conventional MRI. Reduction in $\mathrm{N}$-acetyl-aspartate by proton magnetic spectroscopy in the motor cortex or the brainstem of the patients with ALS is reported with different techniques. Its diagnostic value in clinical assessment is uncertain and remains to be established. Diffusion tensor imaging (DTI) reveals the structural integrity of neuronal fibers, and has great diagnostic promise for ALS. It shows reduced diffusion anisotropy in the cortico-spinal tract with good correlation with physiological index, reflecting UMN pathology. Diffusion tensor tractography allows for visualization and evaluation of corticospinal and cortico-bulbar tract dysfunction individually in patients with ALS. Although many of these new approaches do not yet reach clinical significance, they have been extensively explored in objective evaluation of upper motor function in patients with ALS. Further investigation is needed to determine and to compare the utility of various neurophysiological and neuro-imaging marker [8].

$S$ Budrewicz et al: "Primary lateral sclerosis (PLS) is one of the forms of motor neuron disease (MND), affecting only upper motor neurons. The diagnosis of PLS should be made on different diagnostic criteria, Pringle or Gordon, but it is usually a diagnosis of exclusion. There are no characteristic findings in standard laboratory and electrophysiological assessment. We present details of a 31-year-old woman who had suffered from progressive paraparesis with right-side predominance. Conventional MRI of brain and spinal cord and diffusion tensor imaging (DTI) studies showed Hyperintense lesions in the upper part of the cervical spinal cord at the level C1 in lateral funicules, in the medulla oblongata at the pyramidal decussation and in the midbrain. Brain DTI revealed changes along the cortico-spinal tracts on fractional anisotropy (FA) maps. MRI of the thoracic spinal cord showed in T2-weighted images hyper-intensive regions in the course of the lateral corticospinal tracts. This aided in PLS recognition.

PLS recognition is based on clinical and EMG examination after the exclusion of other diseases, and according to either Pringle's or Gordon's criteria. In our patient conventional brain MRI revealed hyperintense lesions in T2-weighted images along the pyramidal tracts. Similar changes were found in earlier neuro-imaging and neuro-pathological studies in patients with MND. Hyperintensity probably reflects the level of gliosis and demyelination. We did not observe any changes in the cortex of the precentral gyrus and other cortical regions as other authors. Focal decrease in the FA value on brain DTI maps found in the course of cortico-spinal tracts, hyperintensity in T2-weighted images in the lateral cortico-spinal tracts of the thoracic spinal cord indicated axonal degeneration of the pyramidal tracts in our patient.Wang et al. indicated the meaning of changes in posterior limb internal capsule (PLIC) in the diagnosis of PLS. In our case, the DTI showed areas of decreased FA value in PLIC, cerebral peduncles as well as in the spinal cord, mostly the C1 segment. To the best of our knowledge there have been few studies on MRI changes in the spinal cord in patients with different types of MND. The asymmetry of pyramidal tract lesions closely corresponded to the clinical findings (asymmetry of paresis). According to Agosta et al.the DTI could be useful in differential diagnosis between PLS and ALS patients with the highest accuracy of corpus callosum FA values, but they did not perform MRI of the spinal cord.

The authors emphasize the importance of neuro-imaging in PLS recognition. A good quality, high-resolution MRI is able to visualize changes in pyramidal tracts at the level of the spinal cord and DTI study at the level of the brain in PLS patients. This suggests the utility of spinal cord abnormality disclosure as an analytical tool for PLIC changes in MND patients [9].

Bradley R Foerster et al: "ALS is a fatal motor neuron disease for which a precise cause has not yet been identified. Standard CT or MRI evaluation does not demonstrate gross structural nervous system changes in ALS, so conventional neuro-imaging techniques have provided little insight into the pathophysiology of this disease. Advanced neuro-imaging techniques-such as structural MRI, diffusion tensor imaging and proton magnetic resonance spectroscopy-allow evaluation of alterations of the nervous system in ALS. These alterations include focal loss of grey and white matter and reductions in white matter tract integrity, as well as changes in neural networks and in the chemistry, metabolism and receptor distribution in the brain. Given their potential for investigation of both brain structure and function, advanced neuro-imaging methods offer important opportunities to improve diagnosis, guide prognosis, and direct future treatment strategies in ALS. we review the contributions made by various advanced neuroimaging techniques to our understanding of the impact of ALS on 
different brain regions, and the potential role of such measures in biomarker development.

\section{Brainstem}

\section{Diffusion tensor imaging}

One of the first ALS studies to focus on the pyramidal tract in the brainstem reported decreased FA at the level of the pons and medulla in patients versus controls. By contrast, no diseaseassociated differences in FA or MD were detected in these brainstem regions in another study, although they were evident at the level of the cerebral peduncle. Other reports have found reduced FA in the pyramid of the medulla, which correlated with UMN scores.

\section{Proton magnetic resonance spectroscopy}

Decreased NAA:Cr ratios and increased Glx:Cr ratios were detected in the medulla of patients with ALS compared with control individuals. The Glx:Cr ratio showed a significant negative correlation with ALSFRS-R bulbar subscores in patients with bulbar-onset disease. Reductions in brainstem levels of NAA have also been detected in patients with ALS, although no significant changes in Glx were observed.45 A more recent study did not find significant changes in NAA:Cr or NAA:Cho ratios in the medulla or pons in patients with early-onset ALS, although disease duration did show a significant negative correlation with NAA:Cr ratios in the medulla.

A large 18F-FDG PET study found evidence of hyper-metabolism in the midbrain and pons of patients with ALS. Another study showed increased uptake of the PET ligand 11C-L-deprenyl in the pons and supratentorial white matter in ALS, providing support for involvement of astrocytosis in ALS pathogenesis.

Advanced neuro-imaging studies demonstrate structural and metabolic changes in the brainstem in ALS. DTI studies of the brainstem are subject to greater variability than are studies of other brain regions owing to decussation of fibre tracts in this region., significant reductions of FA that correspond to the CST results discussed above have been demonstrated in the brainstem of patients with ALS. Overall, results from $1 \mathrm{H}$-MRS studies provide evidence of neuronal degeneration in the brainstem similar to that in the motor cortex. PET findings support the presence of metabolic, neuroinflammatory and reactive changes in the brainstem in ALS.

\section{Cervical Spinal Cord}

\section{Diffusion tensor imaging}

In ALS, DTI studies have shown reduced FA and increased radial diffusivity in the spinal cord, particularly in the distal cervical cord. cross-sectional spinal cord area was reduced, and FA values correlated with ALSFRS-R scores. Focal atrophy of the spinal cord in ALS correlated with muscle deficits. One study also demonstrated focal FA reductions and increased radial diffusivity in dorsal spinal cord columns, which suggests involvement of spinal sensory pathway in ALS. A longitudinal study demonstrated reductions in spinal cord FA and cross-sectional area and increased MD at 9-month follow-up.92

\section{Magnetic resonance spectroscopy}

MRS studies have focused on the upper cervical cord to minimize the challenges of anatomy and motion artefacts. Reduced NAA:Cr and NAA:myo-inositol ratios have been reported in this region in patients with ALS and in presymptomatic carriers of SOD1 mutations. One study found increased myo-inositol:Cr ratios, which were not confirmed in the other MRS studies. NAA:myo-inositol ratios correlated with FVC127,129 and ALSFRS-R scores, as well as with rates of decline in these scores.

\section{Implications of imaging findings}

Both DTI and MRS studies of the cervical cord in ALS demonstrate structural and metabolite changes that are concordant with those seen in the brain. new advances in neuroimaging techniques will enable the challenges of investigating this important nervous system structure-which is integrally involved in the pathophysiology of ALS- to be addressed.

\section{Brainstem}

Diffusion tensor imaging-One of the first ALS studies to focus on the pyramidal tract in the brainstem reported decreased FA at the level of the pons and medulla in patients versus controls. By contrast, no disease-associated differences in FA or MD were detected in these brainstem regions in another study, although they were evident at the level of the cerebral peduncle. Other reports have found reduced FA in the pyramid of the medulla, which correlated with UMN scores. Proton magnetic resonance spectroscopy-Decreased NAA:Cr ratios and increased Glx:Cr ratios were detected in the medulla of patients with ALS compared with control individuals.The Glx:Cr ratio showed a significant negative correlation with ALSFRS-R bulbar subscores in patients with bulbar-onset disease. Reductions in brainstem levels of NAA have also been detected in patients with ALS, although no significant changes in Glx were observed. A more recent study did not find significant changes in NAA: $\mathrm{Cr}$ or NAA: Cho ratios in the medulla or pons in patients with early-onset ALS, although disease duration did show a significant negative correlation with NAA:Cr ratios in the medulla.

PET -A large 18F-FDG PET study found evidence of hypermetabolism in the midbrain and pons of patients with ALS. Another study showed increased uptake of the PET ligand 11C-L-deprenyl in the pons and supratentorial white matter in ALS, providing support for involvement of astrocytosis in ALS pathogenesis.

Implications of imaging findings-Advanced neuro-imaging studies demonstrate structural and metabolic changes in the brainstem in ALS. DTI studies of the brainstem are subject to greater variability than are studies of other brain regions owing to decussation of fibre tracts in this region. significant reductions of FA that correspond to the CST results discussed above have been demonstrated in the brainstem of patients with ALS. Overall, results from $1 \mathrm{H}-\mathrm{MRS}$ studies provide evidence of neuronal degeneration in the brainstem similar to that in the motor cortex. PET findings 
support the presence of metabolic, neuroinflammatory and reactive changes in the brainstem in ALS.

\section{Cervical Spinal Cord}

\section{Diffusion tensor imaging}

In ALS, DTI studies have shown reduced FA and increased radial diffusivity in the spinal cord, particularly in the distal cervical cord. cross-sectional spinal cord area was reduced, and FA values correlated with ALSFRS-R scores. Focal atrophy of the spinal cord in ALS correlated with muscle deficits. One study also demonstrated focal FA reductions and increased radial diffusivity in dorsal spinal cord columns, which suggests involvement of spinal sensory pathway in ALS. A longitudinal study demonstrated reductions in spinal cord FA and cross-sectional area and increased MD at 9-month follow-up.

\section{Magnetic resonance spectroscopy}

MRS studies have focused on the upper cervical cord to minimize the challenges of anatomy and motion artefacts. Reduced NAA:Cr and NAA:myo-inositol ratios have been reported in this region in patients with ALS and in presymptomatic carriers of SOD1 mutations. 1 study found increased myoinositol: $\mathrm{Cr}$ ratios, which were not confirmed in the other MRS studies. NAA:myoinositol ratios correlated with FVC, and ALSFRS-R scores,as well as with rates of decline in these scores.

\section{Imaging findings}

Both DTI and MRS studies of the cervical cord in ALS demonstrate structural and metabolite changes that are concordant with those seen in the brain. New advances in neuroimaging techniques will enable the challenges of investigating this important nervous system structure-which is integrally involved in the pathophysiology of ALS—-to be addressed [10].

Hernando Rafael et al: These results confirm our previous observations, that the etiology of this disease is of ischemic origin in the intraparenchymal (bulbar pyramids; piramidal decussation; ambiguus nuclei; the ventral respiratory groups or expiratory centers; the $\mathrm{A} 1 / \mathrm{C} 1$ cell groups (related with the arterial pressure); dorsal motor nuclei of the vagus nerves; facial nuclei; hypoglossal nuclei and nucleus of the solitary tracts, especially the ventrolateral portions, also known as the dorsal respiratory groups or inspiratory centers, among others) territory of the AVSAs whose normal average diameter is of $0.75 \mathrm{~mm}$, and/or ASA with an average diameter of $0.80 \mathrm{~mm}$ up to about T1 level. this disease is not neruodegenerative; because it can be cured (mild stage) or improved (moderate or severe stage) through an omental transplantation on the anterior surface of the medulla oblongata. A conclusion contrary to most researchers, who continue to support that ALS is a neruo-degenerative disease, and therefore has not cure.

\section{Operation}

The surgery was performed in 2 stages under general anesthesia: First, supraumbilical laparotomy to remove a segment of omentum and then, cervical laminectomy at the C1-C2 and C5-C6 (only in 9 cases) levels.During the laparotomy, in 36 patientsthe omentum was of poor quality; because this tissue was found thickened, pale and greasy (milky spots), ovoid, rounded, and poorly vascularized. A patient had not major omentum, because he havebeen suffered colon surgery several years ago, for this reason, we obtained almost all the minor omentum.

The medulla oblongata and cervical cord at C5-C6 level, was located as previously described technique. During surgery we found: 1) hypertrophy of the posterior arc of atlas and spinous process of the axis; 2) hypertrophy of the yellow ligament; 3) inestability of the cervical spine; 4) anatomical variants of the V4 segments of the vertebral arteries; 5) moderate to severe atherosclerosis in both V4 segments; 6) absence or unilateral stenosis of the AVSAs, 7) several exsanguinated shorth and long circumferential arteries (arterial branches originated from the AVSAs and/or ASA); 8) several perforating arteries bloodless over the surface of the medulla oblongata; 9) hypotrophy of small nerve roots in the row of IX, X and XI cranial nerves, and 10) hypotrophy of the anterior roots at C5-C6 level. A segment of omentum was placed on the anterior (piramidal decussation and bulbar pyramids), lateral and posterior surface of the medulla oblongata and fixed to the dura mater by its inner face, and into 9 patients of this group, they received an additional segment of omentum on the anterior, lateral and posterior surface of the cervical cord (at C5-C6 level) by spinal ALS. In all cases, the dura matter was laxly faced and not to water seal. 2 patients had transient arterial hypotension during the surgery and no bytransoperative bleeding.

35 patients were extubated in the operating room. 8 patients were extubated in the intermediate care room between 1 and 4 hours after surgery due to weakness in the ventilation. 1 patient remained at intensive care unit by one day, and the other patient, during 3 weeks by respiratory complications. These 10 patients had malnutrition and/or severe ALS. almost all patients left the operating room with nasogastric and Foley tube for 2 and 4 days. The nasogastric tube to receive liquid feed and hydratation. In 2004, based on neurosurgical experiences with omental transplantation,one of us published a hypothesis about the neruodegenerative mechanisms. Hypothesis supported currently by several authors, that the neruo-degenerative process is secondary to the formation of free radicals and oxidative stress in the ischemic area; provoking an imbalance between the oxidant and anti-oxidant (endogenous defenses) systems, in favor of the oxidants. Under normal biochemical and physiological conditions in any area of the brain, the levels of free radicals are controlled by enzymes such as catalase, glutatione peroxidase and various types of superoxide dismutase, among other endogenous defenses (anti-oxidants) which prevent the process of oxidative stress, ie., irreversible damage to intracellular biological molecules such as DNA, RNA, proteins and lipids. In this way in the nervous tissue in ischemia and ischemic penumbra by atherosclerosis and/or the influence of environmental chemicals (cigarette smoking, pesticides and organic solvents, etc), a normal neuron with aerobic respiration, it turn into in other ischemic neurons with anaerobic respiration, 
associated with a decrease of ATP, loss of control of cell membrane and increased free radicals formation. both factors (atherosclerosis and toxic agents) directly or indirectly increases the formation of free radicals, followed by oxidative stress, neurodegeneration and finally, localized or diffuse atrophy. the use of exogenous antioxidants (Resveratrol, Vit A, Vit C and Vit E, etc) and/or the stem cell transplantation by intraspinal or intravenous injection for the treatment of ALS, could be of little utility; because the ischemic areas (intraparenchymal territory of AVSAs and ASA) would not receive these substances nor transplanted stem cells. vascular recanalization through aspirin may allow exogenous antioxidants to enter the ischemic areas. That is, without vascular recanalization or revascularization in the ischemic zones, residual neurons no re-send axons to specific areas already establised, ie., no neuronal regeneration or neurogenesis occurs [11].

Sicong Tu et al: "The cerebellum shows neuro-pathological change in a number of neruo-degenerative conditions where clinical involvement is not the primary feature, including amyotrophic lateral sclerosis (ALS). Whether these changes are associated with disruption to the direct cerebellar tract pathways to the motor cortex and spinal cord in ALS is uncertain. Diffusion tensor imaging was used to examine the integrity of 2 primary cerebellar pathways, the dentato-rubro-thalamo-cortical (DRTC) and spino-cerebellar (SC) tracts. ALS patients with an upper motor neuron (UMN)-predominant phenotype $(n=9)$, were matched to a group with the UMN-only condition primary lateral sclerosis (PLS, $n=10)$ and healthy controls $(n=17)$. Significant alterations across diffusion metrics in the DRTC proximal to the motor cortex were found in both patient groups. PLS patients were found to have an independent diffusion abnormality in the cerebellar region of the DRTC and SC tracts. Disruption to primary cerebellar tracts in PLS is therefore postulated, adding to other markers of its divergent pathogenesis from ALS [12]

Cristina Grande, et al: Gastropod mitochondrial genomes exhibit an unusually great variety of gene orders compared to other metazoan mitochondrial genome such as e.g those of vertebrates. gastropod mitochondrial genomes constitute a good model system to study patterns, rates, and mechanisms of mitochondrial genome rearrangement. this kind of evolutionary comparative analysis requires a robust phylogenetic framework of the group under study, which has been elusive so far for gastropods in spite of the efforts carried out during the last 2 decades. we report the complete nucleotide sequence of 5 mitochondrial genomes of gastropods, and we analyze them together with another ten complete mitochondrial genomes of gastropods currently available in molecular databases in order to reconstruct the phylogenetic relationships among the main lineages of gastropods. Comparative analyses with other mollusk mitochondrial genomes allowed us to describe molecular features and general trends in the evolution of mitochondrial genome organization in gastropods. Phylogenetic reconstruction with commonly used methods of phylogenetic inference (ME, MP, ML, BI) arrived at a single topology, which was used to reconstruct the evolution of mitochondrial gene rearrangements in the group.
4 main lineages were identified within gastropods: Caenogastropoda, Vetigastropoda, Patellogastropoda, and Heterobranchia. Caenogastropoda and Vetigastropoda are sister taxa, as well as, Patellogastropoda and Heterobranchia. The gene order of the Vetigastropoda might represent the ancestral mitochondrial gene order for Gastropoda and we propose that at least 3 major rearrangements have taken place in the evolution of gastropods: one in the ancestor of Caenogastropoda, another in the ancestor of Patellogastropoda, and one more in the ancestor of Heterobranchia [13].

Cristina Grande et al: "Pulmonata includes marine, freshwater and terrestrial gastropods with very different body plans. The monophyly of Pulmonata has been accepted by many authors based on some morphological characters like the streptoneuran inervation of the cephalic tentacles, and the lack of rhinophoric nerve (present in opisthobranchs and pyramidellids). the essential, traditionally accepted morphological synapomorphy of Pulmonata is the presence of a special neuro-secretory system comprising procerebrum and cerebral gland. The procerebrum is formed of small and large neuronal cells, and because it links the peripheral tentacular structures with the central nervous system, an olfactory function has been assumed. The cerebral gland is a neuronal structure associated with the cerebral ganglia. New molecular data reject Pulmonata as a natural group based on both nuclear and mitochondrial data. In this study, we have included representatives of all major lineages within pulmonates (Systelommatophora, Basommatophora, Ellobiidae, and Stylommatophora).

All these lineages independently reject the definition of pulmonates as a natural group in all the performed analyses. Stylommatophora (land snails) is a monophyletic group, in agreement with previous morphological studies, and it is recovered as the sister group to all other heterobranchs studied. Our results provide new insights into land colonization by heterobranch gastropods. The transition to a land lifestyle was accompanied by a variety of refined morphological and physiological modifications. As a result, land snails and slugs constitute a well-defined group of pulmonates with several morphological synapomorphies in the cephalic tentacles, kidney, and central nervous system, as well as in several aspects of their ontogeny. Previous phylogenetic hypotheses had suggested that the transition to land was a rather derived event in the history of pulmonates. Our molecular phylogeny instead supports a different scenario in which gastropod land colonization, and subsequent radiation was an early and significant event in the evolution of Heterobranchia [13].

Yoshihisa Kurita et al: Gastropods are characterized by their asymmetric bodyplan, which develops through a unique ontogenetic process called 'torsion'. Despite several intensive studies, the driving force of torsion remains to be determined. Although torsion was traditionally believed to be driven by contraction of the retractor muscle connecting the foot and the shell, some recent reports cast doubt on that idea. Here, we report that torsion is accompanied by left-right asymmetric cell proliferation in the mantle epithelium in 
the limpet Nipponacmea fuscoviridis. Furthermore, we found that pharmacological inhibition of the transforming growth factor- $\beta$ (TGF- $\beta$ ) signalling pathway, including that of Nodal, blocked torsion. We confirmed that the blocking was brought about through failure of the activation of cell proliferation in the right-hand side of the mantle epithelium, while the retractor muscle apparently developed normally. These results suggest that limpet torsion is driven by left-right asymmetric cell proliferation in the mantle epithelium, induced by the TGF- $\beta$ pathway [14].

Rengasayee Veeraraghavan et al: In addition to the structural substrate, conduction is also modulated by dynamic functional changes. Primarily, these dynamics result from the interplay between the strength of the excitatory impulse (the source) and the electrical load represented by the tissue it must excite (the sink). In the adult canine ventricular myocardium, each myocyte is coupled to an average of $11 \pm 3$ other myocytes. As an activation wavefront spreads through the myocardium, the amount of source available per unit mass of tissue is determined by its excitability, whereas the balance between source and sink is determined by the curvature of the wavefront and its interaction with the architecture of the myocardium: intercellular coupling, fiber orientation, rotational anisotropy, branching tissue geometry. Since local excitability in tissue is dynamically modulated by changes in the shape and duration of action potentials, mismatch between source and sink can arise locally and dynamically, creating a functional substrate for arrhythmogenic conduction defects. Pathophysiological gap junction remodeling and fibrosis can exacerbate source-sink mismatch and thereby the propensity for arrhythmias. For a more detailed discussion of source-sink mismatch, the reader is referred to the in depth review of electrotonic conduction by Drs. A. Kleber and Y. Rudy [15].

PW Nathan et al: "This paper is a clinic-pathological study of the manner in which the spino-thalamic tract crosses the cord from its cells of origin to the opposite anterior and anterolateral funiculi. The introduction of anterolateral cordotomy led to much discussion of the manner in which the spinothalamic tract crosses the cord. There are 2 views: one is that the fibres cross transversely; the other is that they cross diagonally, taking several segments to cross. In this paper we present the evidence that the fibres cross transversely and also theoretical reasons why they cannot be crossing diagonally. The first investigator to make use of anterolateral cordotomy to study the anatomy of the spinal cord was Foerster. He wrote that he had had seven cases with a sensory level at the segment below the incision. He concluded that the fibres cross the cord within one to one-and-a-half segments above their cell bodies and that when this result is not obtained the incision has not been deep enough. Later, Foerster and Gagel published a series of cases in which there were nine autopsies. But they did not make use of this histological material, stating that their conclusions were based on clinical observation and not on `anatomical proof.

Babtchine, working in Leningrad, obtained analgesia and thermanaesthesia up to the level of the incised segment and confirmed Foerster's view. Kuru, who studied with Foerster, had detailed histological material from 10 cases of cordotomy. His conclusions supported Foerster and Gagel. White and colleagues found that an analgesic level to within one segment of the incision was rarely obtained and they concluded that this was due to the diagonal crossing of the fibres. They therefore wrote that their opinion differed from 'that of Foerster and Gagel but conforms with most other observers'. With further evidence, White modified his view, stating that 'the number of segments required for decussation for the pain fibres varies from one individual to another, the fibres requiring only one to 2 segments to cross in some and at least 6 in others'. He reported a case in which the incisions into the cord were at T4 or T5 with hypoalgesia at T5 level. In 1954 he reported 20 cases of cordotomy carried out at $\mathrm{C} 2$; the analgesia was up to the territory of the fifth cranial nerve in 2 of them.

One of the best papers on this subject is that published by White and colleagues in 1956. In this paper, as in many others, the reader needs to distinguish the level of sensory loss obtained immediately after the operation from levels seen when some return of sensibility had taken place. Their paper reported a study of $\sim 300$ upper thoracic cordotomies, in 18 of which there was histological examination of the cord. They concluded that 'the more extensive transections of white matter have resulted in higher and more consistent levels of analgesia'. Bohm reported that, in 28 of 35 cases of unilateral cervical cordotomy, there was complete analgesia extending to one segment below the incision. In 10 of these patients the incision was at C1-2 and in 12 it was between C3 and C4. Šourek concluded from his experience of anterolateral cordotomy that the fibres 'take one to 3 segments to cross the cord'. On reading the literature, one finds that adequate attention has not been given to the relationship between the surgical incision and the fibres passing from the anterior commissure and through the anterior horn. Yet this relationship provides an important clue showing that the fibres cross the cord transversely [16].

Welniarz Q et al: The cortico-spinal tract (CST) plays a major role in cortical control of spinal cord activity. In particular, it is the principal motor pathway for voluntary movements. Here, we discuss: (i) the anatomic evolution and development of the CST across mammalian species, focusing on its role in motor functions; (ii) the molecular mechanisms regulating cortico-spinal tract formation and guidance during mouse development; and (iii) human disorders associated with abnormal CST development. A comparison of CST anatomy and development across mammalian species first highlights important similarities. In particular, most CST axons cross the anatomical midline at the junction between the brainstem and spinal cord, forming the pyramidal decussation. Reorganization of the pattern of CST projections to the spinal cord during evolution led to improved motor skills. Studies of the molecular mechanisms involved in CST formation and guidance in mice have identified several factors that act synergistically to ensure proper formation of the CST at each step of development. Human CST developmental disorders can result in a reduction of the CST, or in guidance defects associated with abnormal CST 
anatomy. These latter disorders result in altered midline crossing at the pyramidal decussation or in the spinal cord, but spare the rest of the CST. Careful appraisal of clinical manifestations associated with CST malformations highlights the critical role of the CST in the lateralization of motor control [17].

Mohamed-Mounir El Mendili et al: The mechanisms underlying the topography of motor deficits in spinal muscular atrophy (SMA) remain unknown. We investigated the profile of spinal cord atrophy (SCA) in SMN1-linked SMA, and its correlation with the topography of muscle weakness. Eighteen SMN1-linked SMA patients type III/V and 18 age/gender-matched healthy volunteers were included. Patients were scored on manual muscle testing and functional scales. Spinal cord was imaged using 3T MRI system. Radial distance (RD) and cord cross-sectional area (CSA) measurements in SMA patients were compared to those in controls and correlated with strength and disability scores.

CSA measurements revealed a significant cord atrophy gradient mainly located between C3 and C6 vertebral levels with a SCA rate ranging from $5.4 \%$ to $23 \%$ in SMA patients compared to controls. RD was significantly lower in SMA patients compared to controls in the anterior-posterior direction with a maximum along $\mathrm{C} 4$ and C5 vertebral levels ( $\mathrm{p}$-values $<10-5$ ). There were no correlations between atrophy measurements, strength and disability scores. Spinal cord atrophy in adult SMN1-linked SMA predominates in the segments innervating the proximal muscles. Additional factors such as neuro-muscular junction or intrinsic skeletal muscle defects may play a role in more complex mechanisms underlying weakness in these patients [18].

Burcu Zeydan et al: "To assess whether cervical spinal cord atrophy heralds the onset of progressive MS.

We studied 34 individuals with radiologically isolated syndrome (RIS) and 31 patients with relapsing-remitting MS (RRMS) age matched to 25 patients within a year of onset of secondary progressive MS (SPMS). 2 raters independently measured (twice per rater) the cervical spinal cord average segmental area (CASA) (mm2) of axial T2-weighted images between C2 and C7 landmarks. The midsagittal T2-weighted image from the end of $\mathrm{C} 2$ to the end of C7 vertebra was used to measure the cervical spine (c-spine) length (mm). Sex, age at cervical MRI, number and location of cervical spinal cord lesions, c-spine length, and diagnoses were analyzed against the outcome measures of CASA and C2 and C7 slice segmental areas.

Intrarater and interrater agreement was excellent (intraclass correlation coefficient $>0.97)$. The CASA area $(p=0.03)$ and C7 area $(p=0.002)$ were smaller in SPMS compared with RRMS. The C2 area $(p=0.027)$, CASA $(p=0.004)$, and C7 area $(p=0.003)$ were smaller in SPMS compared with RIS. The C2 area did not differ between SPMS and RRMS ( $p=0.09)$. The C2 area ( $=0.349)$, CASA $(p=0.136)$, and $C 7$ area $(p=0.228)$ did not differ between RIS and MS (SPMS and RRMS combined). In the multivariable model, $\geq 2$ cervical spinal cord lesions were associated with the $\mathrm{C} 2$ area
( $p=0.008)$, CASA $(p=0.009)$, and C7 area independent of disease course $(p=0.017)$. Progressive disease course was associated with the $\mathrm{C7}$ area independent of the cervical spinal cord lesion number ( $p=0.004)$. Cervical spinal cord atrophy is evident at the onset of progressive MS and seems partially independent of the number of cervical spinal cord lesions" [19].

Hiroto Nakano et al: "We herein describe a 37-year-old man who developed cervical flexion myelopathy 11 years after suffering a cervical spinal cord injury. Cervical magnetic resonance imaging 11 years after the accident demonstrated atrophy and hyperintense lesions at the C6 and C7 levels in the cervical cord with an abnormal alignment of the vertebrae. In the neck flexion position, an anterior shift of the cervical cord was evident. Our patient's condition suggests that an abnormal alignment of the cervical spine and spinal cord injury due to a traumatic accident could be risk factors in the subsequent development of cervical flexion myelopathy [20].

Wheeler-Kingshott CA et al: "This study describes a new technique for Diffusion Tensor Imaging (DTI) that acquires axial (transverse) images of the cervical spinal cord. The DTI images depict axonal fiber orientation, enable quantification of diffusion characteristics along the spinal cord, and have the potential to demonstrate the connectivity of cord white matter tracts. Because of the high sensitivity to motion of diffusion-weighted magnetic resonance imaging and the small size of the spinal cord, a fast imaging method with high in-plane resolution was developed. Images were acquired with a single-shot EPI technique, named ZOOM-EPI (zonally magnified oblique multislice echo planar imaging), which selects localized areas and reduces artefacts caused by susceptibility changes between soft tissue and the adjacent vertebrae. Cardiac gating was used to reduce pulsatile flow artefacts from the surrounding cerebrospinal fluid. Voxel resolution was 1.25 x $1.25 \mathrm{~mm}$ (2) in-plane with 5-mm slice thickness. Both the mean diffusivity (MD) and the fractional anisotropy (FA) indices of the cervical spinal cord were measured. The FA index demonstrated high anisotropy of the spinal cord with an average value of 0.61 +/- 0.05 (highest value of $0.66+/-0.03$ at C3), comparable to white matter tracts in the brain. The diffusivity components parallel and orthogonal to the longitudinal axes of the cord were lambda(parallel) $=(1648+/-123) \times 10(-6) \mathrm{mm}(2) \mathrm{s}(-1)$ and lambda(perpendicular $)$ $=(570+/-47) \times 10(-6) \mathrm{mm}(2) \mathrm{s}(-1)$, respectively. The high axial resolution allowed preliminary evaluation of fiber connectivity using the fast-marching tractography algorithm, which generated traces of fiber paths consistent with the well-known cord anatomy [21].

C Chien et al: Measures for spinal cord atrophy have become increasingly important as imaging biomarkers in the assessment of neuro-inflammatory diseases, especially in neuro-myelitis optica spectrum disorders. The most commonly used method, mean upper cervical cord area, is relatively easy to measure and can be performed on brain MRIs that capture cervical myelon. Measures of spinal cord volume (eg, cervical cord volume or total cord volume) require longer scanning and more complex analysis but are 
potentially better suited as spinal cord atrophy measures. This study investigated spinal cord atrophy measures in a cohort of healthy subjects and patients with aquaporin-4 antibody seropositive neuro-myelitis optica spectrum disorders and evaluated the discriminatory performance of mean upper cervical cord crosssectional area compared with cervical cord volume and total cord volume. Spinal cord atrophy (SCA) can appear after longitudinally extensive transverse myelitis $7 \Downarrow-9$ and has been suggested as an imaging biomarker for disease severity and treatment response in NMOSD. Previous studies have demonstrated that SCA can be associated with longer disease duration and more severe disability and can potentially indicate poor prognosis in NMOSD.

The SCA measurement most commonly used is the mean upper cervical cord area (MUCCA). It is measured as the crosssectional area of the spinal cord at the level between the $\mathrm{C} 2$ and C3 vertebrae. Although longitudinally extensive transverse myelitis predominantly affects the cervical and upper thoracic spinal cord, the lower thoracic and lumbar cord in patients with NMOSD is often affected as well. Hence, the main disadvantage of MUCCA is that though it can be easily obtained and measured, it covers only a very small fraction of the spinal cord. quantification of the total cord volume (TCV) is theoretically beneficial for a more precise assessment of SCA [22].

Christopher P. Gilmore et al: "Our finding of White Matter atrophy restricted to the upper regions of the spinal cord is consistent with previous work using this postmortem material. These studies have demonstrated that whole-cord atrophy and atrophy of the lateral and posterior WM columns are not observed in the lower cord. Although the cervical cord has a predilection for demyelinating WM lesions, tissue loss within WM lesions does not have a significant influence on local cord Atrophy. the distribution of WM lesions within the cord may still influence the pattern of atrophy through the effects of wallerian degeneration. For example, the preservation of ascending WM tracts in the lower cord may reflect the paucity of WM lesions in this region and explain, in part, the normal WM volume in the lower cord.

Our results suggest that spinal cord atrophy in MS is purely due to WM volume loss. Our observation of preserved GM volume is a notable one, highlighting important differences between the GM of the spinal cord and the GM structures of the brain. A greater understanding of the mechanisms of GM disease is required to explain these differences [23].

Kanehiro Fujiyoshi et al: In spinal cord injury, axonal disruption results in motor and sensory function impairment. The evaluation of axonal fibers is essential to assess the severity of injury and efficacy of any treatment protocol, but conventional methods such as tracer injection in brain parenchyma are highly invasive and require histological evaluation, precluding clinical applications. Previous advances in magnetic resonance imaging technology have led to the development of diffusion tensor tractography (DTT) as a potential modality to perform in vivo tracing of axonal fibers. The properties and clinical applications of DTT in the brain have been reported, but technical difficulties have limited DTT studies of the spinal cord. In this study, we report the effective use of DTT to visualize both intact and surgically disrupted spinal long tracts in adult common marmosets. To verify the feasibility of spinal cord DTT, we first performed DTT of postmortem marmosets. DTT clearly illustrated spinal projections such as the corticospinal tract and afferent fibers in control animals and depicted the severed long tracts in the injured animals. Histology of the spinal cords in both control and injured groups were consistent with DTT findings, verifying the accuracy of DTT. We also conducted DTT in live marmosets and demonstrated that DTT can be performed in live animals to reveal in vivo nerve fiber tracing images, providing an essential tool to evaluate axonal conditions in the injured spinal cord. Taken together, these findings demonstrate the feasibility of applying DTT to preclinical and clinical studies of spinal cord injury [24].

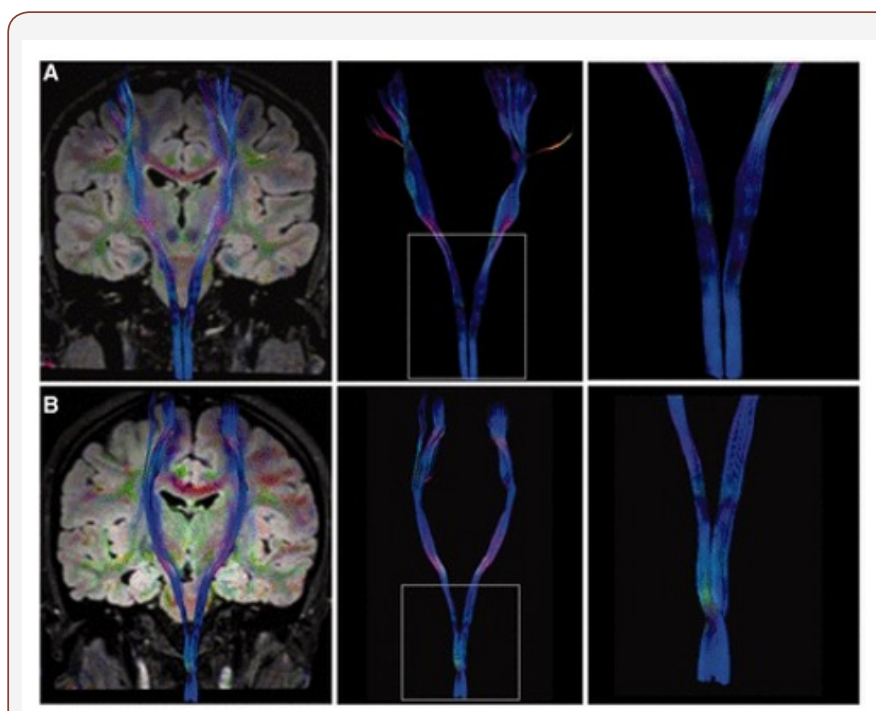

Figure 6: 3.0 T MRI DTI tractography, (A) missing decussation of pyramids, with strictly unilateral non-crossing cortico-spinal tracts, and (B) a control subject, with normal crossing cortico-spinal tracts. (from reference n. 25).

Pedro Brandão et al: "Sir, We have read with great interest the article published by Gallea et al., in 2013 issue of Brain, regarding the pathophysiological basis of the rare disease named congenital mirror movements, that might serve as a model to recognize new aspects of bimanual motor control. We have recently seen a patient with congenital mirror movements and have coincidently studied this disorder using a clinical, neuro-physiological and neuroimaging protocol similar to those used by Gallea et al.. This case was presented as a video session at the 5th Meeting of the Movement Disorders Scientific Department of the Brazilian Academy of Neurology, in August 2013, but has not yet been published. Our patient is a 32-year-old right-handed male, with synkinetic distal movements of distal limbs since early childhood, with stable course, and no other movement disorders or neurological abnormalities. The involuntary movements led him to labour impairment as he works as a postman and has difficulties in performing bimanual tasks such as writing on a clipboard or handling a mobile telephone 
or keyboard. Interestingly, a late acquisition of running abilities was his only neuro-developmental delay; his parents reporting a preference for jumping instead of running in early childhood [which may be similar to the Kanga mice hopping gait, with spontaneous mutation in the deleted in colorectal carcinoma (Dcc) gene]. His clinical manifestations were classified in the Woods and Teuber Mirror Movements Scale as 3 of 4 (strong and sustained repetitive mirror movements)" [25] (Figure 6).

Agosta F et al: "To explore structural and functional changes of the brain and cervical cord in patients with amyotrophic lateral sclerosis (ALS) due to mutation in the superoxide dismutase (SOD1) gene compared with sporadic ALS. Twenty patients with SOD1 ALS, 11 with sporadic ALS, and 33 healthy controls underwent clinical evaluation and brain MRI. Cortical thickness analysis, diffusion tensor MRI of the cortico-spinal tracts (CST) and corpus callosum and resting-state functional connectivity were performed. Patients with ALS also underwent cervical cord MRI to evaluate cord crosssectional area and magnetization transfer ratio (MTR).

Patients with SOD1 ALS showed longer disease duration and slower rate of functional decline relative to those with sporadic ALS. No cortical thickness abnormalities were found in patients with ALS compared with controls. Fractional anisotropy showed that sporadic ALS patients had significant CST damage relative to both healthy controls ( $p=0.001-0.02)$ and SOD1-related ALS ( $p=0.05)$, although the latter showed alterations that were intermediate between controls and sporadic ALS. Functional hyper connectivity of the motor cortex in the sensorimotor network was observed in patients with sporadic ALS relative to controls. Conversely, patients with SOD1 ALS showed lower cord cross-sectional area along the whole cervical cord relative to those with sporadic ALS ( $\mathrm{p}<0.001)$. No cord MTR differences were found between patient groups.

Patients with SOD1 ALS showed cervical cord atrophy relative to those with sporadic ALS and a relative preservation of brain motor structural and functional networks. Neurodegeneration in SOD1 ALS is likely to occur primarily in the spinal cord. An objective and accurate estimate of spinal cord damage has potential in the future assessment of preventive SOD1 ALS therapies [26].

$S$ Budrewicz et al: "Primary lateral sclerosis (PLS) is one of the forms of motor neuron disease (MND), affecting only upper motor neurons. The diagnosis of PLS should be made on different diagnostic criteria, for example, Pringle or Gordon, but it is usually a diagnosis of exclusion. There are no characteristic findings in standard laboratory and electrophysiological assessment. We present details of a 31-year-old woman who had suffered from progressive paraparesis with right-side predominance. Conventional MRI of brain and spinal cord and diffusion tensor imaging (DTI) studies showed Hyperintense lesions in the upper part of the cervical spinal cord at the level C1 in lateral funicules, in the medulla oblongata at the pyramidal decussation and in the midbrain. Brain DTI revealed changes along the cortico-spinal tracts on fractional anisotropy (FA) maps. MRI of the thoracic spinal cord showed in T2-weighted images hyper-intensive regions in the course of the lateral cortico-spinal tracts. This aided in PLS recognition [27].

Ephron S. Rosenzweiget al: "The bilaterally-projecting and terminating CST architecture may influence spontaneous CNS recovery after CNS injury. For example, direct input from each motor cortex to both sides of the spinal cord may contribute to recovery after unilateral stroke in humans. Reduced inhibition of the intact cortex (across the corpus callosum) could unmask preexisting connections between the intact cortex and the affected (ipsilateral) spinal cord. Moreover, bilateral terminal arbors arising from single CST axons could provide an anatomical basis for mirror movements of the hands and forelimbs that are sometimes observed after unilateral stroke. Nirkko et al. examined latencies of motor potentials evoked by transcranial magnetic stimulation, and determined that mirror movements were at least partially caused by direct innervation of both sides of the spinal cord by branching CST axons (though the authors could not determine the rostrocaudal level of branching). Thus, the present findings identify candidate anatomical mechanisms that could underlie some forms of plasticity observed after CNS injury.

These studies were performed as part of the development of a primate model of spinal cord injury (SCI). Our description of the bilateral nature of the primate CST and of the presence of spinally-decussating CST pathways highlights a potential target for therapeutic intervention after incomplete SCI. SCI research has generally focused on regeneration of transected axons, but more recently there has been recognition that enhanced sprouting of spared axons may be a useful target for promoting functional recovery. The basis for this shift is at least threefold. spinal cord injury and other central nervous system or peripheral nerve lesions can induce spontaneous sprouting of nearby systems into territory denervated by the lesion, it has proven easier to experimentally enhance sprouting of spared axons than to stimulate regeneration of transected axons. most humans who have sustained spinal cord injury suffer complete loss of function below the lesion yet exhibit anatomical sparing of white matter on the spinal cord periphery ; this spared tissue could be targeted for therapeutic enhancement. The latter point is particularly important in light of the present findings because the CST, critical for fine motor control in humans, may retain bilateral projections with only unilateral or asymmetric tissue sparing [28].

Accorsdin Herbert L. Fred: "In the United States, ALS affects approximately 1 in 50,000 people per year. About 90\% of cases are sporadic, 5 including the occasional appearance in couples and in clusters. The remaining cases are familial,6 inherited as a Mendelian trait.Patients lose control of voluntary movement, speech, swallowing, and respiration. Death is typical within 2 to 3 years after onset. Some patients, die after 1 or 2 months, and others might live for 10 or more years with the support of artificial feeding and respiration. Others who have died of ALS include U.S. Vice President Henry A. Wallace, U.S. Senator Jacob K. Javits, U.S. Army General Maxwell D. Taylor, actor David Niven, boxer Ezzard Charles, 
jazz musician Charles Mingus, Jr., and another Hall of Fame baseball player, pitcher Jim “Catfish" Hunter." [29].

Chiò A et al: "The cause of amyotrophic lateral sclerosis (ALS) is still unknown. A possible relationship between ALS and sport participation has been supposed, but never definitely demonstrated. We studied a cohort of 7325 male professional football players engaged by a football team from the Italian First or Second Division in the period 1970-2001. ALS cases were identified using different concurrent sources. Standardized morbidity ratios (SMRs) were calculated. During the 137,078 person-years of follow-up, 5 ALS cases were identified (mean age of onset, 43.4 years). 3 cases had a bulbar onset, significantly more than expected $(P=0.003)$. Since the number of expected cases was 0.77 , the overall SMR was 6.5 [95\% confidence interval (CI), 2.1-15.1]. The SMR was significantly increased for an ALS onset before 49 years, but not for older subjects. A significant increase of the SMR was found in the periods 1980-1989 and 1990-2001, whereas no ALS case was found in the 1970-1979 period. A dose-response relationship between the duration of professional football activity and the risk of ALS was found $(>5$ years, $15.2,95 \% \mathrm{CI}, 3.1-44.4$; $<$ or $=5$ years, $3.5,95 \% \mathrm{CI}$, $0.4-12.7)$. Our findings seem to indicate that playing professional football is a strong risk factor for ALS [31].

Lehman EJ et al: "To analyze neruo-degenerative causes of death, specifically Alzheimer disease (AD), Parkinson disease, and amyotrophic lateral sclerosis (ALS), among a cohort of professional football players.

This was a cohort mortality study of 3,439 National Football League players with at least 5 pension-credited playing seasons from 1959 to 1988. Vital status was ascertained through 2007. For analysis purposes, players were placed into 2 strata based on characteristics of position played: nonspeed players (linemen) and speed players (all other positions except punter/kicker). External comparisons with the US population used standardized mortality ratios (SMRs); internal comparisons between speed and nonspeed player positions used standardized rate ratios (SRRs).

Overall player mortality compared with that of the US population was reduced (SMR 0.53, 95\% confidence interval [CI] 0.48-0.59). Neruo-degenerative mortality was increased using both underlying cause of death rate files (SMR 2.83, 95\% CI 1.36-5.21) and multiple cause of death (MCOD) rate files (SMR 3.26, 95\% CI 1.90-5.22). Of the neruo-degenerative causes, results were elevated (using MCOD rates) for both ALS (SMR 4.31, 95\% CI 1.73-8.87) and AD (SMR 3.86, 95\% CI 1.55-7.95). In internal analysis (using MCOD rates), higher neruo-degenerative mortality was observed among players in speed positions compared with players in nonspeed positions (SRR 3.29, 95\% CI 0.92-11.7). The neruo-degenerative mortality of this cohort is 3 times higher than that of the general US population; that for 2 of the major neruo-degenerative subcategories, AD and ALS, is 4 times higher. These results are consistent with recent studies that suggest an increased risk of neruo-degenerative disease among football players [31].
Ingre et al: Athleticism, chronic traumatic encephalopathy, and physical exercise "Historically, the most famous ALS patient was Lou Gehrig, the renowned US baseball player. Several studies have demonstrated increased risk of ALS among football or soccer players, other athletes, and individuals who engage in vigorous physical activity, but inconsistent results have also been reported. Strenuous physical activity, repeated head injuries, use of illicit performance-enhancing drugs, or chemicals used to treat football fields have all been discussed as potential explanations for such risk elevations. Chronic traumatic encephalopathy, a newly defined neruo-degenerative disease, often resulting from repeated head injuries, has been proposed as the underlying reason or the "correct" diagnosis for ALS cases observed among professional athletes and perhaps also among military veterans. Different levels of physical exercise (professional versus recreational) may have very different biological effects on neuro-degeneration. This is in line with previous findings of an increased risk of ALS among professional football players, although not among high school players. Similarly, a large European case-control study showed a 51\% lower risk of ALS for organized sport, but a 59\% higher risk of ALS for professional sport. Further efforts to disentangle the different exposure patterns involved in professional sports as compared with recreational sports will be needed to better understand these findings. Although the hypothesis that athleticism contributes to ALS is intriguing, caution should be exercised in interpreting these findings, given the fact that the vast majority are based on small numbers of ALS cases.

Head trauma: Early case-control studies reported a significant association between history of head trauma and ALS. Aiming to rule out the possibilities of recall bias and reverse causality (ie, trauma as a result, rather than a cause, of ALS), later studies generally used more objective assessment of head trauma history and excluded traumas experienced during the years immediately before the diagnosis of ALS. Severe head traumas that were hospitalized were not associated with a higher risk of ALS in Sweden. A possible association of ALS with milder head traumas, perhaps specifically with repeatedly experienced mild traumas, has not been thoroughly addressed.

Neuro-inflammation: Since the earliest pathological changes in ALS appear to occur in axons, dendrites, and synapses, studies of the relationship between inflammatory conditions around the motor unit and ALS may shed light on the pathological development of ALS. Clinically, early symptoms of ALS can be difficult to differentiate from symptoms of other inflammatory neuromuscular diseases such as myositis, myasthenia gravis, Guillain-Barré syndrome, and multiple sclerosis. Due to the difficulties in determining the correct diagnosis, misdiagnosis may be an explanation for any higherthan-expected co-occurrence of ALS and inflammatory diseases. Interestingly, ALS and multiple sclerosis were reported to co-occur in individuals with the C9ORF72 repeat expansion, suggesting some biological overlaps between ALS and autoimmune/inflammatory diseases. apart from several reports of cases diagnosed with both ALS and with some of the conditions above, few studies have addressed this issue. 
Occupations: Workers in various occupations with seemingly disparate exposures have been reported to be potentially at altered risk of ALS, including athletes, carpenters, cockpit workers, construction workers, electrical workers, farm workers, hairdressers, house painters, laboratory technicians, leather workers, machine assemblers, medical service workers, military workers, nurses, power production plant workers, precision metal workers, programmers, rubber workers, shepherds, tobacco workers, veterinarians, and welders. These occupations potentially involve work exposures to chemicals, pesticides, metals, and electromagnetic fields (EMF). common denominators among these different occupations are not easily identified. Electric occupation, electric shock, and electromagnetic field ALS has been associated with "electrical" occupations, especially welding. Magnetic fields, electrical fields, contact currents, micro-shocks, and both perceptible and imperceptible electric shocks all contribute to occupational exposure to extremely low frequency EMF. The reported association of ALS with EMF is generally weaker than that with electrical occupations. Evidence is not yet available to distinguish whether electric shocks or exposure to EMF underlies the association between electrical occupation and ALS. A metaanalysis suggested that there might be a slight but statistically significant increase in ALS risk among people with job descriptions related to relatively high levels of EMF exposure [32].

Troy Shinbrot et al: "Many vertebrate motor and sensory systems "decussate" or cross the midline to the opposite side of the body. The successful crossing of millions of axons during development requires a complex of tightly controlled regulatory processes. Because these processes have evolved in many distinct systems and organisms, it seems reasonable to presume that decussation confers a significant functional advantage - the nature of this advantage is not understood. Surprisingly, even in some small networks of only 100-1,000 neurons, on the order of the number of neural connections in common insect tracts, decussation can reduce the rate of erroneous connections by over an order of magnitude. we predict that 3D networks larger than this, and so spatially dependent tracts of animals only as complex as insects, must decussate in order to function reliably. It is interesting to note in this context that many proteins involved in developmental decussation, for example, netrins, slits, and UNCs, are highly evolutionarily conserved and are seen in the most primitive of insects, worms, and other invertebrates as well as in vertebrates. The presence of an elementary decussated tract (the ventral tract) even in C. Elegans (a nematode) suggests, that the evolutionary advantage of decussation may have predated the creation of detailed visual organs or limbs, contrary to the dominant paradigm described in.

These simulations indicate a plausible mechanism by which decussated systems could have evolved using only random mutations in independent systems whose fitness depends on wiring accuracy. the simulations demonstrate that there is a dramatic quantitative advantage in robustness of decussated networks against miswiring events when compared with the alternative ipsilateral configuration. the findings suggest that decussated pathways may be prevalent in vertebrate nervous systemsbecause decussation minimizes pathfinding errors and genetic information content required, and provides significant evolutionary advantages. But even in small networks of only 100-1,000 neurons, on the order of the number of neural connections in common insect tracts, decussation can reduce the rate of erroneous connections by over an order of magnitude" [33].

Experimental project (hypotesys to verify): In order to verify the connection between some anatomic peculiarity like decussatio pyramidi and cervical district and some neruo-degenerative condition we submit to the researcher a new methods to classify the imaging report under a strictly topographic way in order to show in the different neurologic disease of spinal cord (cervical vs other presentation). All this imaging from different techniques must added in this new database to verify the \% of Lesions in the global spinal cords. In this kind of approach a statistical method make possible to verify if exist a correlation between this anatomic peculiarity and a weakness of nervous tract in spinal cord pyramids.

\section{Discussion}

To start this discussion is possible to observe that in some heart pathology like ipertrophia the anatomy of the fibre of miocyte and also to their fiber orientation can result in some kind of artimia. This fact can produce interferences in physiology like metabolic, electrical conduction and other? The same other similar situation can produce the same effect. What happen when high amount of nervous motor fibres decussate in spinal cord medullary portion? And related some condition like head micro-trauma or other pathologic movens? Or in particular weakness of nervous systems? (in condition like ALS, spinal atrophy and other neurologic or inflamatory, metabolic, blood irroration and traumatic). From evolutionary science is possible to see that in some gasteropods there is a torsion of internal organs and also of the visceral nerve.

This kind of invertebrates (pulmonated) colonized land form sea and need to develop new motor system different from acquatic environment. To prevent deidration they developed spiralic shell and this caused a torsion of internal organ. Is possible to consider decussatio piramidy and ancestral anatomic modify in superior vertebrates? What is the real scope of this cross of motor nerve? only a selective process ? But is interesting to observe finally the consequences that this kind of anatomic peculiarity can have in spinal cord phisio-pathlogy.

This kind of cross of motor fiber in some stressing condition can cause effect like: electrical interference, metabolic and catabolic kinetics and other properties? Is also easily possible to observe that even if all humans have a pancreas only some subjects develop a Type I diabetes (according a genetic profile as endogenous factor). So also an anatomic peculiarity present in all human in some situation can cause different effect. In example what happen in micro-trauma on head of football players or in some baseball players? And Related ALS pathology is interesting to observe the weakness of some neuronal circuits (pyramidal - motor), length can 
influence this? and related decussatio pyramid ? can be considered an Stressing condition?

Related spinal cord disorder cause can be of various origin: like

1) compressive

2) vascular

3) inflammatory

4) infectious

5) development

6) metabolic

7) Other: transverse myelitis, trauma (hyper-extension, pull, tears)

In amyotrophic lateral sclerosis is possible to verify that spinal cord lesion are often also at cervical level and the same related multiple sclerosis an also in other condition. In MS: "the cervical cord has a predilection for demyelinating [23]. Observing some phenomena from other science like geology and engineering When an old wall present various rift often crashes down following this intrinsic new weakness. (the various vector of force Produce a global response). According $\mathrm{CHIO}^{\prime}$ et al “Our findings seem to indicate that playing professional football is a strong risk factor for ALS [30]. And Lehman "for 2 of the major neruo-degenerative subcategories, AD and ALS, is 4 times higher [31]. Due to bipedal position humans present often lumbar vertebres problems because over used in an unnatural position (in example vs quadruped locomotion) the same this evolutionary position (bipedal) can influence cervical vertebres and cervical spinal cord and related neuron phisio-pathology (repeated head micro-trauma and injuries, hyper-extention, pull, tears and other).

\section{Conclusion}

Related to the reference findings presented in this review and research work is crucial to submit to the researcher a new hypotesis related the anatomic decussatio pyramid and cervical tract in global physio-metabolic status of Spinal cord. In same condition like repeated head micro-trauma, or in particular weakness of motor neuron this anatomic conformation can produce un unbalance that can aggravate this situation. (see the higher frequencies related cervical tract vs other in spinal cord). A fact to be take in consideration even if is a normal anatomic conformation. The experimental project proposed make possible to verify any connection between an anatomic peculiarity (decussatio pyramidi, cervical spinal cord tract) and an increased weakness of the neuronal tracts. So in neuro- degenerative spinal cord disease is relevant to observe the global topography of the lesions to verify if some tract are more involved vs other to better understand the real reason. The evolutionary approach make possible to better understand the global process under a new light.

\section{Clarifications}

This work is produced without any diagnostic or therapeutic intent, only to submit to the researcher New hypothesis.

\section{Acknowledgement}

None

\section{Conflict of Interest}

No conflict of interest.

\section{References}

1. Louise R Page (2006) Modern insights on gastropod development: Reevaluation of the evolution of a novel body plan. Integrative and Comparative Biology 46(2): 134-143.

2. Shuichi Shigeno, Paul LR Andrews, Giovanna Ponte, Graziano Fiorito (2018) Cephalopod Brains: An Overview of Current Knowledge to Facilitate Comparison With Vertebrates. Front Physiol 9: 952.

3. Larsson M (2013) The optic chiasm: a turning point in the evolution of eye/hand coordination. Front Zool 10(1): 41

4. Kinsbourne M (2013) Somatic twist: a model for the evolution of decussation. Neuropsychology 27(5): 511-515.

5. De Lussanet MH, Osse JW (2015) Decussation as an axial twist: A comment on Kinsbourne (2013). Neuropsychology 29(5): 713-714.

6. Shinbrot T, Young W (2008) Why Decussate? Topological Constraints on 3D Wiring. Anat Rec (Hoboken) 291: 1278-1292.

7. Marc Christopher Emos, Sanjeev Agarwal (2018) Neuroanatomy, Upper Motor Neuron Lesion.

8. Iwata NK (2007) Objective markers for upper motor neuron involvement in amyotrophic lateral sclerosis. Brain Nerve 59(10): 1053-1064.

9. S Budrewicz, P Szewczyk, K Slotwinski, M Koszewicz (2015) Symptoms of degeneration of the pyramidal tracts in conventional magnetic resonance imaging and diffusion tensor imaging in a young woman with primary lateral sclerosis. J Postgrad Med 61(3): 206-208.

10. Bradley R Foerster, Robert C Welsh, Eva L Feldman (2013) 25 years of neuro-imaging in amyotrophic lateral sclerosis. Nat Rev Neurol 9(9): 513-524.

11. Rafael H, David JO, Vilca AS (2017) Etiology and treatment of amyotrophic lateral sclerosis. Am J Neurodegener Dis 6(1): 1-8.

12. Sicong Tu, Ricarda AL Menke, Kevin Talbot, Matthew C Kiernan, Martin R Turner (2019) Cerebellar tract alterations in PLS and ALS. ORCID Icon Short Report.

13. Grande C, Templado J, Zardoya R (2008) Evolution of gastropod mitochondrial genome arrangements. BMC Evol Biol 8: 61.

14. Yoshihisa Kurita, Hiroshi Wada (2011) Evidence that gastropod torsion is driven by asymmetric cell proliferation activated by TGF- $\beta$ signaling. Biol Lett 7(5): 759-762.

15. Veeraraghavan R, Gourdie RG, Poelzing S (2014) Mechanisms of cardiac conduction: a history of revisions. Am J Physiol Heart Circ Physio 306(5): H619-H627.

16. Nathan PW, Smith M, Deacon P (2001) The crossing of the spinothalamic tract. Brain 124(4): 793-803.

17. Welniarz Q Dusart I, Roze E (2017) The cortico-spinal tract: Evolution, development, and human disorders. Dev Neurobiol 77(7): 810-829.

18. El Mendili MM, Lenglet T, Stojkovic T, Behin A, Guimarães Costa R (2016) Cervical Spinal Cord Atrophy Profile in Adult SMN1-Linked SMA. PLoS One 11(4): e0152439.

19. Burcu Zeydan, MD, Xinyi Gu, MD, Elizabeth J. Atkinson, MS, B. Mark Keegan, et al. (2018) Cervical spinal cord atrophy, an early marker of progressive MS onset. Neurol Neuroimmunol Neuroinflamm 5(2): e435.

20. Hiroto Nakano, Kenji Sakai, Kazuo Iwasa, Masahito Yamada (2017) Cervical Flexion Myelopathy Eleven Years after a Cervical Spinal Cord Injury. intern Med 56(16): 2213-2215.

21. Wheeler-Kingshott CA, Hickman SJ, Parker GJ, Ciccarelli O, Symms MR, et al. (2002) Investigating cervical spinal cord structure using axial diffusion tensor imaging. Neuroimage 16(1): 93-102. 
22. Gilmore CP, DeLuca GC, Bö L, Owens T, Lowe J, et al. (2005) Spinal Cord Atrophy in Multiple Sclerosis caused by White Matter Volume Loss. Arch Neurol 62(12): 1859-1862.

23. Fujiyoshi K, Yamada M, Nakamura M, Yamane J, Katoh H, et al. (2007) In Vivo Tracing of Neural Tracts in the Intact and Injured

24. Spinal Cord of Marmosets by Diffusion Tensor Tractography. The Journal of Neuroscience 27(44): 11991-11998.

25. Pedro Brandão, Cassio Jovem, Joaquim Pereira Brasil-Neto, Carlos Tomaz, Maxime Descoteaux, et al. (2014) Congenital mirror movements: lack of decussation of pyramids. Brain 137(8): e292.

26. Agosta F, Spinelli EG, Marjanovic IV, Stevic Z, Pagani E, et al. (2018) Unraveling ALS due to SOD1 mutation through the combination of brain and cervical cord MRI. Neurology 90(8): e707-e716.

27. Budrewicz S, Szewczyk P, Slotwinski K, Koszewicz M (2015) Symptoms of degeneration of the pyramidal tracts in conventional magnetic resonance imaging and diffusion tensor imaging in a young woman with primary lateral sclerosis. J Postgrad Med 61(3): 206-208.
28. Rosenzweig ES, Brock JH, Culbertson MD, Lu P, Moseanko R, et al. (2009) Extensive Spinal Decussation and Bilateral Termination of Cervical Cortico-spinal Projections in Rhesus Monkeys. J Comp Neurol 513(2): 151-163.

29. Herbert L. Fred (2017) Lou Gehrig and Ed Todd: Greatness Interrupted. Tex Heart Inst J 44(6): 383-384.

30. Chiò A, Benzi G, Dossena M, Mutani R, Mora G (2005) Severely increased risk of amyotrophic lateral sclerosis among Italian professional football players. Brain 128(Pt 3): 472-476.

31. Lehman EJ, Hein MJ, Baron SL, Gersic CM (2012) Neruo-degenerative causes of death among retired National Football League players. Neurology 79(19): 1970-1974.

32. Ingre C, Roos PM, Piehl F, Kamel F, Fang F (2015) Review Risk factors for amyotrophic lateral sclerosis. Clin Epidemiol 7: 181-193.

33. Shinbrot T, Young W (2008) Why Decussate? Topological Constraints. Anat Rec (Hoboken) 291: 1278-1292. 